\title{
A CASE STUDY IN BIGRADED COMMUTATIVE ALGEBRA
}

\section{INTRODUCTION}

The purpose of this chapter is to illustrate how bigraded commutative algebra differs from the classical graded case and to indicate some of the theoretical tools needed to understand free resolutions in the bigraded case. Bigraded commutative algebra is a special case of multigraded commutative algebra, which is in turn an instance of toric (or polytopal) algebra.

Generalizing classical concepts of algebraic geometry or commutative algebra to the multigraded setting is a very active area of current research; a good overview of the area is given in Sturmfels. For instance, bigraded Castelnuovo-Mumford regularity is studied in Aramova-Crona-De Negri, Römer, and Hoffman-Wang, and generalizations to the multigraded case are investigated in Maclagan-Smith. Another important topic is the study of the toric Hilbert scheme, which parameterizes the set of all ideals having the same multigraded Hilbert function as some fixed toric ideal. References for this are Peeva-Stillman1, Peeva-Stillman2, Maclagan-Thomas, and Stillman-Sturmfels-Thomas. In a different direction, there is also the study of zero-dimensional schemes in the multigraded setting, which began in Giuffrida-Maggioni-Ragusa, and is studied further in Há-Van Tuyl, Van Tuyl, and Sidman-Van Tuyl.

In this chapter we consider the bigraded commutative algebra of the polynomial ring $R=k[x, y, z, w]$, where $k$ is algebraically closed, and $x, y$ have degree $(1,0)$ and $z, w$ have degree $(0,1)$. We will write $R_{m, n}$ for the graded piece of $R$ in degree $(m, n)$. Our main object of study is the ideal $I \subset R$ generated by three bihomogeneous polynomials

$$
p_{0}, p_{1}, p_{2} \in R_{2,1}
$$

assuming that the $p_{i}$ have no common zeros on $\mathbb{P}^{1} \times \mathbb{P}^{1}$.

In the singly graded case, it is well-known that if three homogeneous polynomials in $k[s, t, u]$ have no common zeros on $\mathbb{P}^{2}$, then their Koszul complex is a free resolution of the ideal they generate. We will see that life is more complicated (and more interesting!) in the bigraded case. In particular, for the above polynomials $p_{0}, p_{1}, p_{2}$, we will prove that

- the Koszul complex of $p_{0}, p_{1}, p_{2}$ is never exact, and

- there are two possible minimal free resolutions of the ideal $I$.

In each section of the chapter, we highlight the tools from commutative algebra that are used. The techniques include regular sequences, depth, spectral sequences, vanishing theorems, determinants, the Segre embedding, 
resultants, and mapping cones. Our main results are the determination of the Hilbert function and minimal free resolution of the ideal $I$. In the last section of the chapter, we outline some connections to the implicitization problem in geometric modeling.

\section{Preliminary Analysis of the Koszul Complex}

Tools: Regular sequences, depth, the Buchsbaum-Eisenbud criterion

1.1. The Koszul Complex. In this section, we will assume that $p_{0}, p_{1}, p_{2} \in$ $R$ have degree $\left(d_{1}, d_{2}\right), d_{1}, d_{2}>0$, and no common zeros on $\mathbb{P}^{1} \times \mathbb{P}^{1}$. This gives the Koszul complex

$$
\begin{aligned}
& 0 \rightarrow R\left(-3 d_{1},-3 d_{2}\right) \stackrel{\left[\begin{array}{c}
p_{2} \\
-p_{1} \\
p_{0}
\end{array}\right]}{\longrightarrow} R\left(-2 d_{1},-2 d_{2}\right)^{3} \stackrel{\left[\begin{array}{ccc}
p_{1} & p_{2} & 0 \\
-p_{0} & 0 & p_{2} \\
0 & -p_{0} & -p_{1}
\end{array}\right]}{\longrightarrow} \\
& R\left(-d_{1},-d_{2}\right)^{3} \stackrel{\left[\begin{array}{lll}
p_{0} & p_{1} & p_{2}
\end{array}\right]}{\longrightarrow} R \rightarrow 0 .
\end{aligned}
$$

1.2. The Failure of Exactness. Our first observation is that (1.1) is not exact. This follows by analyzing the zero locus of $p_{0}, p_{1}, p_{2}$ in $\mathbb{A}^{4}$. Since $d_{1}, d_{2}>0$, we know that $p_{0}, p_{1}, p_{2}$ vanish on

$$
\mathbf{V}(x z, x w, y z, y w)=\left(\mathbb{A}^{2} \times\{0\}\right) \cup\left(\{0\} \times \mathbb{A}^{2}\right) \subset \mathbb{A}^{4} .
$$

Furthermore, $p_{0}, p_{1}, p_{2}$ can't vanish simultaneously outside this variety since they have no common zeros on $\mathbb{P}^{1} \times \mathbb{P}^{1}$. Thus $I=\left\langle p_{0}, p_{1}, p_{2}\right\rangle$ has a twodimensional zero locus in $\mathbb{A}^{4}$, i.e, $\operatorname{codim}(I)=2$.

Now assume that the Koszul complex is exact. Then:

- $p_{0}, p_{1}, p_{2}$ form a regular sequence Eisenbud, Thm. 17.6], so that $\operatorname{depth}(I)=3$.

- $R$ is Cohen-Macaulay Eisenbud, Prop. 18.9], so that $\operatorname{codim}(I)=$ $\operatorname{depth}(I)$.

It follows that $\operatorname{codim}(I)=3$, which contradicts the previous paragraph. Thus (1.1) is not exact.

1.3. A More Careful Analysis. There is more to say, for (1.1) still has some exactness. Since $I$ is the image of $R\left(-d_{1},-d_{2}\right)^{3} \rightarrow R$, we can replace (1.1) by

$$
0 \rightarrow R\left(-3 d_{1},-3 d_{2}\right) \rightarrow R\left(-2 d_{1},-2 d_{2}\right)^{3} \rightarrow R\left(-d_{1},-d_{2}\right)^{3} \rightarrow I \rightarrow 0 .
$$

Lemma 1.1. The complex (1.2) is exact except at $R\left(-d_{1},-d_{2}\right)^{3}$.

Proof. We will give two proofs here and a third in the next section. For the first proof, we use a more detailed version of the relation between depth and the Koszul complex. Recall from above that $\operatorname{depth}(I)=\operatorname{codim}(I)=2$. If we denote the Koszul complex (1.1) by

$$
K: 0 \longrightarrow K_{0} \stackrel{\varphi_{0}}{\longrightarrow} K_{1} \stackrel{\varphi_{1}}{\longrightarrow} K_{2} \stackrel{\varphi_{2}}{\longrightarrow} K_{3} \longrightarrow 0,
$$


then Eisenbud, Thm. 17.4] tells us that

$$
H_{j}(K)=0, j<r \text {, and } H_{r}(K) \neq 0 \Longrightarrow \operatorname{depth}(I)=r .
$$

In particular, we must have $H_{0}(K)=H_{1}(K)=0$, since otherwise $I$ would have depth 0 or 1 . Then we are done since $R\left(-d_{1},-d_{2}\right)^{3} \rightarrow I$ is onto.

For second proof, we apply the Buchsbaum-Eisenbud criterion Eisenbud, Thm. 20.9] to the complex

$$
0 \longrightarrow K_{0} \stackrel{\varphi_{0}}{\longrightarrow} K_{1} \stackrel{\varphi_{1}}{\longrightarrow} K_{2} .
$$

The Fitting ideals $I_{i}$ Eisenbud, Sect. 20.2] of $\varphi_{0}$ and $\varphi_{1}$ are computed using the $i \times i$ minors of these maps. One easily computes that

$$
I_{1}\left(\varphi_{0}\right)=I, \quad I_{2}\left(\varphi_{0}\right)=\{0\}
$$

and

$$
I_{2}\left(\varphi_{1}\right)=I^{2}, \quad I_{3}\left(\varphi_{1}\right)=\{0\},
$$

so that $\varphi_{0}$ has rank 1 and $\varphi_{1}$ has rank 2. Furthermore, $I^{2}$ and $I$ have the same depth since $\sqrt{I^{2}}=\sqrt{I}$ [Eisenbud, Cor. 17.8]. Using $\operatorname{depth}(I)=2$, we obtain

$$
\operatorname{depth}\left(I_{1}\left(\varphi_{0}\right)\right)=\operatorname{depth}\left(I_{2}\left(\varphi_{1}\right)\right)=2 .
$$

Thus the hypotheses of the Buchsbaum-Eisenbud criterion are satisfied, so that (1.3) is exact. As above, the lemma follows.

\section{Describing the Failure of Exactness}

Tools: Sheaf cohomology, spectral sequences, Čech cohomology

2.1. The Syzygy Module. From now on, we will assume that $p_{0}, p_{1}, p_{2} \in$ $R$ have degree $(2,1)$ and don't vanish simultaneously on $\mathbb{P}^{1} \times \mathbb{P}^{1}$. Recall that the syzygy module $\operatorname{Syz}(p)=\operatorname{Syz}\left(p_{0}, p_{1}, p_{2}\right)$ is defined by the exact sequence

$$
0 \longrightarrow \operatorname{Syz}(p) \longrightarrow R(-2,-1)^{3} \stackrel{\left[\begin{array}{lll}
p_{0} & p_{1} & p_{2}
\end{array}\right]}{\longrightarrow} I \longrightarrow 0
$$

Then the Koszul complex gives a map

$$
R(-4,-2)^{3} \longrightarrow \operatorname{Syz}(p)
$$

which is not surjective by Lemma 1.1. Syzygies in the image of this map are called Koszul syzygies, and those not in the image are non-Koszul.

2.2. The Sheaf-Theoretic Koszul Complex. We will use sheaf cohomology and spectral sequences to give an abstract description of the non-Koszul syzygies (explicit descriptions will appear in later sections). Our strategy will be to examine the Koszul complex (1.2) (with $\left(d_{1}, d_{2}\right)=(2,1)$ ) one degree at a time. In degree $(m, n)$, (1.2) gives the complex

$$
0 \longrightarrow R_{m-6, n-3} \longrightarrow R_{m-4, n-2}^{3} \longrightarrow R_{m-2, n-1}^{3} \longrightarrow I_{m, n} \longrightarrow 0,
$$

which is exact except at $R_{m-2, n-1}^{3}$ by Lemma 1.1 . 
We begin with the sheaf-theoretic version of the Koszul complex. Let $\mathcal{O}:=\mathcal{O}_{\mathbb{P}^{1} \times \mathbb{P}^{1}}$ and recall that $\mathcal{O}(m, n)$ is the line bundle on $\mathbb{P}^{1} \times \mathbb{P}^{1}$ with the property that

$$
H^{0}\left(\mathbb{P}^{1} \times \mathbb{P}^{1}, \mathcal{O}(m, n)\right)=R_{m, n} .
$$

The Koszul complex of $p_{0}, p_{1}, p_{2}$ is

$$
0 \rightarrow \mathcal{O}(-6,-3) \rightarrow \mathcal{O}(-4,-2)^{3} \rightarrow \mathcal{O}(-2,-1)^{3} \rightarrow \mathcal{O} \rightarrow 0,
$$

so that tensoring by $\mathcal{O}(m, n)$ gives

$0 \rightarrow \mathcal{O}(m-6, n-3) \rightarrow \mathcal{O}(m-4, n-2)^{3} \rightarrow \mathcal{O}(m-2, n-1)^{3} \rightarrow \mathcal{O}(m, n) \rightarrow 0$.

We write this complex as

$$
0 \rightarrow \mathcal{F}_{0}(m, n) \rightarrow \mathcal{F}_{1}(m, n) \rightarrow \mathcal{F}_{2}(m, n) \rightarrow \mathcal{F}_{3}(m, n) \rightarrow 0 .
$$

The key point is that (2.2) is exact. To see why, recall that for sheaves on $\mathbb{P}^{1} \times \mathbb{P}^{1}$, exactness is equivalent to being exact at every point $P \in \mathbb{P}^{1} \times \mathbb{P}^{1}$. Since $p_{0}, p_{1}, p_{2}$ don't vanish simultaneously at $P$, they generate the unit ideal in the local ring $\mathcal{O}_{\mathbb{P}^{1} \times \mathbb{P}^{1}, P}$. By Eisenbud, Prop. 17.14], it follows that the Koszul complex is exact at $P$.

2.3. The Spectral Sequences. As explained in Griffiths-Harris, pp. 445446], the complex $\mathcal{F}_{\bullet}(m, n)$ has a hypercohomology

$$
\mathbb{H}^{\bullet}\left(\mathbb{P}^{1} \times \mathbb{P}^{1}, \mathcal{F}_{\bullet}(m, n)\right)
$$

and two spectral sequences

$$
\begin{aligned}
& { }^{\prime} E_{2}^{p, q}(m, n)=H^{p}\left(\mathbb{P}^{1} \times \mathbb{P}^{1}, \mathcal{H}^{q}\left(\mathcal{F}_{\bullet}(m, n)\right)\right) \Longrightarrow \mathbb{H}^{\bullet}\left(\mathbb{P}^{1} \times \mathbb{P}^{1}, \mathcal{F}_{\bullet}(m, n)\right) \\
& { }^{\prime \prime} E_{1}^{p, q}(m, n)=H^{q}\left(\mathbb{P}^{1} \times \mathbb{P}^{1}, \mathcal{F}_{p}(m, n)\right) \quad \Longrightarrow \mathbb{H}^{\bullet}\left(\mathbb{P}^{1} \times \mathbb{P}^{1}, \mathcal{F}_{\bullet}(m, n)\right) .
\end{aligned}
$$

Since the Koszul complex (2.2) is exact, we have $\mathcal{H}^{q}\left(\mathcal{F}_{\bullet}(m, n)\right)=0$ for all $q$. Thus the first spectral sequence proves the standard fact that the hypercohomology of a exact complex is trivial.

It follows that the second spectral sequence becomes

$$
{ }^{\prime \prime} E_{1}^{p, q}(m, n)=H^{q}\left(\mathbb{P}^{1} \times \mathbb{P}^{1}, \mathcal{F}_{p}(m, n)\right) \Longrightarrow 0,
$$

where the differential $d_{1}$ is induced by the maps in the Koszul complex.

2.4. Consequences of the Spectral Sequence. To examine this spectral sequence, we set $H^{i}(m, n):=H^{i}\left(\mathbb{P}^{1} \times \mathbb{P}^{1}, \mathcal{O}(m, n)\right)$. Then the spectral sequence becomes

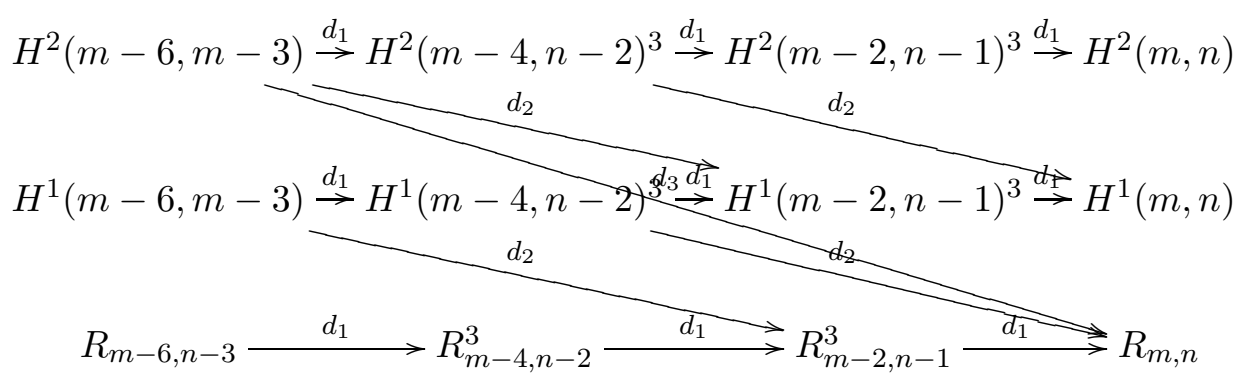


where the ${ }^{\prime \prime} E_{1}^{p, q}(m, n)$ terms are shown. This looks messy but makes it easy to prove the following lemma.

Lemma 2.1. For any $(m, n)$ we have an exact sequence

$$
0 \longrightarrow R_{m-6, n-3} \longrightarrow R_{m-4, n-2}^{3} \longrightarrow S y z(p)_{m, n} \longrightarrow{ }^{\prime \prime} E_{2}^{0,1}(m, n) \longrightarrow 0
$$

where

$$
{ }^{\prime \prime} E_{2}^{0,1}(m, n)=\operatorname{ker}\left(d_{1}: H^{1}(m-6, n-3) \longrightarrow H^{1}(m-4, n-2)^{3}\right)
$$

and $d_{1}$ is induced by the corresponding map in the Koszul complex.

Proof. Converging to 0 means that ${ }^{\prime \prime} E_{\infty}^{p, q}(m, n)=0$ for all $p, q$. First consider $(p, q)=(1,0)$, which corresponds to ${ }^{\prime \prime} E_{1}^{1,0}(m, n)=R_{m-4, n-2}^{3}$ in the above diagram. All differentials $d_{r}, r \geq 2$ that land at or originate from this position must vanish. It follows that

$$
0={ }^{\prime \prime} E_{\infty}^{1,0}(m, n)={ }^{\prime \prime} E_{2}^{1,0}(m, n)=\operatorname{ker}\left(d_{1}\right) / \operatorname{im}\left(d_{1}\right) .
$$

This proves exactness at $R_{m-4, n-2}^{3}$, and exactness at $R_{m-6, n-3}$ is proved similarly. (Note that this gives a third proof of Lemma 1.1] as promised.)

Next consider ${ }^{\prime \prime} E_{1}^{2,0}(m, n)=R_{m-2, n-1}$ and ${ }^{\prime \prime} E_{1}^{0,1}(m, n)=H^{1}(m-6, n-3)$. Here, we have to worry about $d_{2}$, but all higher differentials vanish. It follows that

$$
0={ }^{\prime \prime} E_{\infty}^{2,0}(m, n)={ }^{\prime \prime} E_{3}^{2,0}(m, n) \quad \text { and } \quad 0={ }^{\prime \prime} E_{\infty}^{0,1}(m, n)={ }^{\prime \prime} E_{3}^{0,1}(m, n) .
$$

However, the only way for these " $E_{3}$ terms to vanish is for the map

$$
d_{2}:{ }^{\prime \prime} E_{2}^{0,1}(m, n) \longrightarrow{ }^{\prime \prime} E_{2}^{2,0}(m, n)
$$

to be an isomorphism. Since

$$
\begin{aligned}
& { }^{\prime \prime} E_{2}^{0,1}(m, n)=\operatorname{ker}\left(d_{1}\right) / \operatorname{im}\left(d_{1}\right)=\operatorname{ker}\left(d_{1}\right) \\
& { }^{\prime \prime} E_{2}^{2,0}(m, n)=\operatorname{ker}\left(d_{1}\right) / \operatorname{im}\left(d_{1}\right)=\operatorname{Syz}(p)_{m, n} / \operatorname{im}\left(d_{1}\right),
\end{aligned}
$$

the lemma follows immediately.

This lemma shows that ${ }^{\prime \prime} E_{2}^{0,1}(m, n)$ gives a precise measure of the nonKoszul syzygies. In Section 3 we will use vanishing theorems for sheaf cohomology to compute the size of " $E_{2}^{0,1}(m, n)$ for almost all $(m, n)$.

2.5. Description of $d_{2}$. We will next unveil, in our special case, the spectral sequence machinery that produces a non-Koszul syzygy in degree $(m, n)$, out of an element $\varphi$ in the kernel of the map $d_{1}: H^{1}(m-6, n-3) \rightarrow$ $H^{1}(m-4, n-2)$. The isomorphism

$$
d_{2}: \operatorname{ker}\left(d_{1}\right) \simeq \operatorname{Syz}(p)_{m, n} / \operatorname{im}\left(d_{1}\right) \subset R_{m-2, n-1}^{3} / \operatorname{im}\left(d_{1}\right)
$$

from (2.3) can be explained as follows. 
Consider a Leray covering $\mathcal{U}=\left(U_{i}\right)$ of $\mathbb{P}^{1} \times \mathbb{P}^{1}$, for instance the affine covering given by the four open sets

$$
\begin{aligned}
& U_{1}=\{x \neq 0, z \neq 0\}, U_{2}=\{y \neq 0, z \neq 0\} \\
& U_{3}=\{x \neq 0, w \neq 0\}, U_{4}=\{y \neq 0, w \neq 0\} .
\end{aligned}
$$

Then $H^{1}(m-6, n-3)$ is the Cech cohomology of this cover, so that $\varphi$ is given by a collection of sections $\varphi_{i j}$ of $\mathcal{O}(m-6, n-3)$ in $U_{i} \cap U_{j}$, with $\delta\left(\varphi_{i j}\right)=0$. Since $d_{1}$ comes from the Koszul map, the condition $d_{1}(\varphi)=0$ is equivalent to the existence of sections $\left(\alpha_{i}^{0}, \alpha_{i}^{1}, \alpha_{i}^{2}\right)$ of $\mathcal{O}(m-4, n-2)^{3}$ in each $U_{i}$, such that

$$
p_{k} \cdot \varphi_{i j}=\alpha_{i}^{k}-\alpha_{j}^{k}, \quad \forall i, j, k .
$$

Then, since obviously $p_{k} p_{\ell} \varphi_{i j}=p_{\ell} p_{k} \varphi_{i j}$ for all $i, j, k, \ell$, one easily sees that

$$
p_{\ell} \alpha_{i}^{k}-p_{k} \alpha_{i}^{\ell}=p_{\ell} \alpha_{j}^{k}-p_{k} \alpha_{j}^{\ell}, \quad \forall i, j, k .
$$

This defines a global section $\psi_{k \ell}$ of $\mathcal{O}(m-2, n-1)$. Let $\psi:=\left(\psi_{12},-\psi_{02}, \psi_{01}\right)$. It is straightforward to verify that this is a syzygy, i.e., that the equality $p_{0} \psi_{12}-p_{1} \psi_{02}+p_{2} \psi_{01}=0$ holds.

However, the syzygy $\psi \in \operatorname{Syz}(p)_{m, n}$ is not well-defined, since it depends on the choice of $\alpha_{i}^{k}$ in (2.4). Given another choice

$$
p_{k} \cdot \varphi_{i j}=\beta_{i}^{k}-\beta_{j}^{k}, \quad \forall i, j, k,
$$

we get $\psi^{\prime} \in \operatorname{Syz}(p)_{m, n}$. Comparing this equation to (2.4), we see that $\beta_{i}^{k}-$ $\alpha_{i}^{k}=\beta_{j}^{k}-\alpha_{j}^{k}$ for all $i, j, k$. Thus we have a global section $\gamma^{k}=\beta_{i}^{k}-\alpha_{i}^{k}$ of $\mathcal{O}(m-4, n-2)$. We leave it as an exercise for the reader to verify that $\psi^{\prime}=\psi+d_{1}(\gamma)$, where $\gamma:=\left(-\gamma^{2}, \gamma^{1},-\gamma^{0}\right)$. It follows that the class $[\psi]$ of $\psi$ modulo the image of $d_{1}$ is well-defined. A more substantial exercise is to prove that $d_{2}(\varphi)=[\psi]$ using the Cech description of the spectral sequence of a complex given in Griffiths-Harris, pp. 445-446].

\section{A Picture of the Syzygy Module}

Tools: the Künneth formula, the cohomology of line bundles on $\mathbb{P}^{1}$

3.1. The Degree of a Syzygy. We defined $\operatorname{Syz}(p)$ to be the kernel of the map

$$
R(-2,-1)^{3} \stackrel{\left[\begin{array}{lll}
p_{0} & p_{1} & p_{2}
\end{array}\right]}{\longrightarrow} I .
$$

It follows that

$$
\operatorname{Syz}(p)_{m, n} \subset R(-2,-1)_{m, n}^{3}=R_{m-2, n-1}^{3} .
$$

This shows that syzygies in $\operatorname{Syz}(p)_{m, n}$ are represented by vectors of polynomials of degree $(m-2, n-1)$. It will be important to keep this in mind in the discussion that follows. 
3.2. Vanishing Cohomology. In Lemma 2.1 we saw that the non-Koszul syzygies are determined by

$$
{ }^{\prime \prime} E_{2}^{0,1}(m, n)=\operatorname{ker}\left(d_{1}: H^{1}(m-6, n-3) \rightarrow H^{1}(m-4, n-2)^{3}\right) .
$$

In order to compute this, we first need to compute $H^{1}(m, n)$. We do this as follows.

Lemma 3.1. If either $m, n \geq-1$ or $m, n \leq-1$, then $H^{1}(m, n)=0$.

Proof. Let $\pi_{i}: \mathbb{P}^{1} \times \mathbb{P}^{1} \rightarrow \mathbb{P}^{1}$ be projection onto the $i$ th factor. Then

$$
\mathcal{O}(m, n)=\pi_{1}^{*} \mathcal{O}_{\mathbb{P}^{1}}(m) \times \pi_{2}^{*} \mathcal{O}_{\mathbb{P}^{1}}(n) .
$$

The Künneth formula Dieudonné-Grothendieck, Thm. 6.7.8] implies that $H^{1}(m, n)$ is isomorphic to

$$
H^{0}\left(\mathbb{P}^{1}, \mathcal{O}_{\mathbb{P}^{1}}(m)\right) \otimes H^{1}\left(\mathbb{P}^{1}, \mathcal{O}_{\mathbb{P}^{1}}(n)\right) \oplus H^{1}\left(\mathbb{P}^{1}, \mathcal{O}_{\mathbb{P}^{1}}(m)\right) \otimes H^{0}\left(\mathbb{P}^{1}, \mathcal{O}_{\mathbb{P}^{1}}(n)\right) .
$$

Furthermore, we know that $H^{0}\left(\mathbb{P}^{1}, \mathcal{O}_{\mathbb{P}^{1}}(k)\right)=0$ for $k \leq-1$, and the perfect pairing

$$
H^{0}\left(\mathbb{P}^{1}, \mathcal{O}_{\mathbb{P}^{1}}(-k-2)\right) \times H^{1}\left(\mathbb{P}^{1}, \mathcal{O}_{\mathbb{P}^{1}}(k)\right) \longrightarrow H^{1}\left(\mathbb{P}^{1}, \mathcal{O}_{\mathbb{P}^{1}}(-2)\right) \simeq k
$$

described in Hartshorne Thm. 5.1 of Ch. III] implies that $H^{1}\left(\mathbb{P}^{1}, \mathcal{O}_{\mathbb{P}^{1}}(k)\right)=$ 0 for $k \geq-1$. The lemma now follows easily.

Note that (3.2) is Serre Duality for $\mathbb{P}^{1}$ (see Hartshorne, Thm. 7.1 of Ch. III]). We next apply the lemma to " $E_{2}^{0,1}(m, n)$.

Proposition 3.2. If $m \geq 5, n \geq 2$ or $m \leq 5, n \leq 2$, then ${ }^{\prime \prime} E_{2}^{0,1}(m, n)=0$. Furthermore,

$$
\begin{aligned}
\operatorname{dim}_{k}{ }^{\prime \prime} E_{2}^{0,1}(m, 1)=\operatorname{dim}_{k} H^{1}(m-6,-2) & = \begin{cases}m-5 & m \geq 6 \\
0 & m \leq 5\end{cases} \\
\operatorname{dim}_{k}{ }^{\prime \prime} E_{2}^{0,1}(3, n)=\operatorname{dim}_{k} H^{1}(-3, n-3) & = \begin{cases}2(n-2) & n \geq 3 \\
0 & n \leq 2\end{cases} \\
\operatorname{dim}_{k}{ }^{\prime \prime} E_{2}^{0,1}(4, n)=\operatorname{dim}_{k} H^{1}(-2, n-3) & = \begin{cases}n-2 & n \geq 3 \\
0 & n \leq 2 .\end{cases}
\end{aligned}
$$

Proof. If $m \geq 5, n \geq 2$ or $m \leq 5, n \leq 2$, then $H^{1}(m-6, n-3)=0$ by Lemma 3.1. Then ${ }^{\prime \prime} E_{2}^{0,1}(m, n)=0$ follows by (3.1). To study " $E_{2}^{0,1}(m, 1)$, first observe that $H^{1}(m-4,-1)=0$ for all $m$ by Lemma 3.1. Hence

$$
\operatorname{dim}_{k}{ }^{\prime \prime} E_{2}^{0,1}(m, 1)=\operatorname{dim}_{k} H^{1}(m-6,-2)=\operatorname{dim}_{k} H^{0}\left(\mathbb{P}^{1}, \mathcal{O}_{\mathbb{P}^{1}}(m-6)\right),
$$

where the first equality uses (3.1) and the second uses the Künneth formula and $H^{1}\left(\mathbb{P}^{1}, \mathcal{O}_{\mathbb{P}^{1}}(-2)\right) \simeq k$. Then we are done since

$$
\operatorname{dim}_{k} H^{0}\left(\mathbb{P}^{1}, \mathcal{O}_{\mathbb{P}^{1}}(m-6)\right) \simeq \operatorname{dim}_{k} k[x, y]_{m-6}=m-5 .
$$

The other assertions are similar though slightly more complicated. We leave the details as an exercise. 
3.3. The Picture. For a given graded piece $\operatorname{Syz}(p)_{m, n}$ of the syzygy module, there are three things that could happen:

- the graded piece could be zero, or

- it could be nonzero but consist only of Koszul syzygies, or

- it could contain a non-Koszul syzygy.

Because $p_{0}, p_{1}, p_{2}$ have no common zeros on $\mathbb{P}^{1} \times \mathbb{P}^{1}$, we can predict in advance which of these possibilities occurs for $\operatorname{Syz}(p)_{m, n}$ for most $(m, n)$. Here is the precise result.

Proposition 3.3. The graded pieces $\operatorname{Syz}(p)_{m, n}$ of the syzygy module can be described as follows:

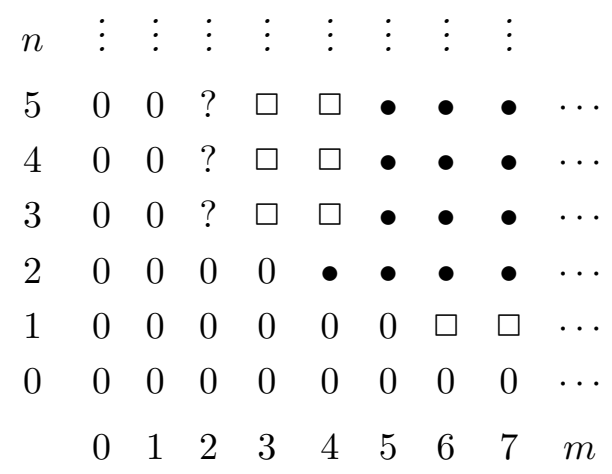

where in position $(m, n)$,

0 means that $\operatorname{Syz}(p)_{m, n}=0$.

- means that $\operatorname{Syz}(p)_{m, n} \neq 0$ and is generated by the Koszul syzygies.

$\square$ means that $\operatorname{Syz}(p)_{m, n}$ contains non-Koszul syzygies. The exact number is computed in Proposition 3.2.

? means that we don't yet know what is going on.

Proof. When $m \leq 1$ or $n=0$, we clearly get zero since elements of $\operatorname{Syz}(p)_{m, n}$ are represented by polynomials of degree $(m-2, n-1)$.

If $m=3$ or $n=1$, then Lemma 2.1 implies that $\operatorname{Syz}(p)_{m, n}={ }^{\prime \prime} E_{2}^{0,1}(m, n)$ for these degrees. The corresponding zeros and boxes follow from (3.3).

For $m=4$, (3.3) implies that ${ }^{\prime \prime} E_{2}^{0,1}(4,2)=0$ and $" E_{2}^{0,1}(4, n) \neq 0$ for $n \geq 3$. Then Lemma 2.1 explains the bullet at position $(4,2)$ and the boxes that lie above it.

Proposition 3.2 tells us that ${ }^{\prime \prime} E_{2}^{0,1}(m, n)=0$ when $m \geq 5$ and $n \geq 2$. Then the corresponding bullets follow from Lemma 2.1.

Finally, " $E_{2}^{0,1}(2,2)=0$ by Proposition 3.2 . But the proposition gives no information about " $E_{2}^{0,1}(2, n)=0$ for $n \geq 3$. Hence the question marks.

In (3.4), the generators of the Koszul syzygies lie in the bullet closest to the origin, at position $(4,2)$. To get some insight into the question marks, consider the lowest question mark, at position $(2,3)$. Then $\operatorname{Syz}(p)_{2,3}=$ 
${ }^{\prime \prime} E_{2}^{0,1}(2,3)$ since there are no Koszul syzygies in this degree. One easily computes that

$$
\operatorname{dim}_{k} H^{1}(-4,0)=3 \quad \text { and } \quad \operatorname{dim}_{k} H^{1}(-2,1)=2 .
$$

Thus $\operatorname{Syz}(p)_{2,3}={ }^{\prime \prime} E_{2}^{0,1}(2,3)=\operatorname{ker}\left(d_{2}: H^{1}(-4,0) \rightarrow H^{1}(-2,1)^{3}\right)$ is the kernel of a map from $k^{3}$ to $k^{6}$. As we will soon see, there are two possibilities for its dimension.

3.4. Exactness Modulo $B$-Torsion. Let $B:=\langle x z, x w, y z, y w\rangle$. If we think of the Koszul complex of sheaves on $\mathbb{A}^{4}$, then we get

$$
0 \longrightarrow \mathcal{O}_{\mathbb{A}^{4}} \longrightarrow \mathcal{O}_{\mathbb{A}^{4}}^{3} \longrightarrow \mathcal{O}_{\mathbb{A}^{4}}^{3} \longrightarrow \mathcal{O}_{\mathbb{A}^{4}} \longrightarrow 0,
$$

where the maps are given by the matrices in (1.1). We showed in Section 1 that $\mathbf{V}\left(p_{0}, p_{1}, p_{2}\right)=\mathbf{V}(B)$ since $p_{0}, p_{1}, p_{2}$ have no common zeros on $\mathbb{P}^{1} \times \mathbb{P}^{1}$. Hence the above complex is exact outside $\mathbf{V}(B)$.

It follows that when we take global sections, the resulting sequence is exact modulo $B$-torsion. (We leave the proof as an exercise for the reader.) Since the sequence of global sections is the Koszul complex (1.1), we conclude that the Koszul complex is exact modulo $B$-torsion.

In particular, every syzygy becomes Koszul by multiplying by elements of $B^{\ell}$ for sufficiently large $\ell$. Since the generators of $B$ have degree $(1,1)$, this is easy to see in the picture (3.4), for multiplying by an element of degree $(1,1)$ moves up and to the right one unit. Looking at the picture, it should be clear that multiplying by $B^{3}$ makes every syzygy Koszul.

Finally, we note that the idea of being exact up to $B$-torsion plays an important role in the concept of toric regularity described in Maclagan-Smith.

\section{The Question Marks}

Tools: the Segre embedding, planes in $\mathbb{P}^{5}$, line bundles on $\mathbb{P}^{1}$

4.1. The Segre Embedding. Before we can study the question marks in (3.4), we need to set up some notation. Elements of $R_{2,1}$ will be written

$$
a x^{2} z+b x y z+c y^{2} z+d x^{2} w+e x y w+f y^{2} w .
$$

We will identify $\mathbb{P}\left(R_{2,1}\right)$ with $\mathbb{P}^{5}$. Since $p_{0}, p_{1}, p_{2} \in R_{2,1}$ are linearly independent (otherwise they would have a common zero on $\mathbb{P}^{1} \times \mathbb{P}^{1}$ ), they determine a plane

$$
W(p):=\mathbb{P}\left(\operatorname{Span}\left(p_{0}, p_{1}, p_{2}\right)\right) \subset \mathbb{P}^{5} .
$$

Reducible polynomials in $R_{2,1}$ can factor in two ways according to the multiplication maps

$$
R_{2,0} \times R_{0,1} \rightarrow R_{2,1} \quad \text { and } \quad R_{1,1} \times R_{1,0} \rightarrow R_{2,1} .
$$

The first will prove to be relevant to our situation. It gives the Segre embedding

$$
\mathbb{P}^{2} \times \mathbb{P}^{1}=\mathbb{P}\left(R_{2,0}\right) \times \mathbb{P}\left(R_{0,1}\right) \rightarrow \mathbb{P}^{5}=\mathbb{P}\left(R_{2,1}\right) .
$$


We will denote the image of this map as

$$
Y \subset \mathbb{P}^{5} .
$$

It is well-known (and easy to verify using Macaulay 2) that $Y \subset \mathbb{P}^{5}$ has degree 3 , dimension 3 , and is defined by the vanishing of the $2 \times 2$ minors of the matrix

$$
\left[\begin{array}{lll}
a & b & c \\
d & e & f
\end{array}\right]
$$

where $a, \ldots, f$ are homogeneous coordinates of $\mathbb{P}^{5}$. This is clear if we write the elements of $R_{2,1}$ in the form

$$
\left(a x^{2}+b x y+c y^{2}\right) z+\left(d x^{2}+e x y+f y^{2}\right) w,
$$

since the vanishing of the maximal minors of the matrix (4.1) is equivalent to the quadratic forms $a x^{2}+b x y+c y^{2}$ and $d x^{2}+e x y+f y^{2}$ being proportional.

4.2. The Two Cases. Our assumption that $p_{0}, p_{1}, p_{2} \in R_{2,1}$ have no common zeros on $\mathbb{P}^{1} \times \mathbb{P}^{1}$ is very strong. However, as we will now prove, it is not strong enough to determine the question marks in (3.4).

Theorem 4.1. Let $W(p)$ and $Y$ be as above. Then:

(1) If $W(p) \cap Y$ is finite, then $\operatorname{Syz}(p)_{2, n}=0$ for all $n \geq 3$.

(2) If $W(p) \cap Y$ is infinite, then $\operatorname{Syz}(p)_{2, n}$ has dimension $n-2$ for all $n \geq 3$.

Remark 4.2. Since $Y \subset \mathbb{P}^{5}$ has codimension 2 and $W(p)$ is a plane, $W(p) \cap$ $Y$ is finite when $W(p)$ is generic. This observation leads to the following terminology:

- $p_{0}, p_{1}, p_{2}$ are generic if $W(p) \cap Y$ is finite. In this case, the question marks in (3.4) are all zeros.

- $p_{0}, p_{1}, p_{2}$ are non-generic if $W(p) \cap Y$ is infinite. In this case, the question marks are all boxes (since syzygies in $\operatorname{Syz}(p)_{2, n}$ are nonKoszul).

The proof given below will show that in the non-generic case, $W(p) \cap Y$ is a smooth conic in $W$. We will also see that a suitable parametrization of this conic gives an element of $\operatorname{Syz}(p)_{2,3}$ whose multiples generate $\operatorname{Syz}(p)_{2, n}$ for all $n \geq 3$.

Proof. The graded piece $\operatorname{Syz}(p)_{2, n}$ consists of syzygies $A_{0} p_{0}+A_{1} p_{1}+A_{2} p_{2}=$ 0 , where $A_{0}, A_{1}, A_{2}$ have degree $(0, n-1)$. Suppose that $\operatorname{Syz}(p)_{2, n} \neq 0$ for some $n \geq 3$. Pulling out the GCD, we can write this syzygy as the triple

$$
h(z, w)\left(A_{0}(z, w), A_{1}(z, w), A_{2}(z, w)\right),
$$

where $A_{0}, A_{1}, A_{2}$ are coprime. It is easy to see that $\left(A_{0}, A_{1}, A_{2}\right)$ is also a syzygy on $p_{0}, p_{1}, p_{2}$. Furthermore, $A_{0}, A_{1}, A_{2}$ are nonconstant by (3.4).

Now consider the map $\varphi: \mathbb{P}^{1} \rightarrow W(p)$ defined by

$$
(s, t) \mapsto p_{s, t}:=A_{0}(s, t) p_{0}+A_{1}(s, t) p_{1}+A_{2}(s, t) p_{2} .
$$


Since $A_{0}, A_{1}, A_{2}$ are nonconstant with $\operatorname{GCD}\left(A_{0}, A_{1}, A_{2}\right)=1$, this is defined on all of $\mathbb{P}^{1}$ and the image is a curve.

However, $p_{s, t}(x, y, z, w)$ has the interesting property that $p_{s, t}(x, y, s, t)$ is given by

$$
A_{0}(s, t) p_{0}(x, y, s, t)+A_{1}(s, t) p_{1}(x, y, s, t)+A_{2}(s, t) p_{2}(x, y, s, t),
$$

which vanishes identically since $\left(A_{0}, A_{1}, A_{2}\right)$ is a syzygy on $p_{0}, p_{1}, p_{2}$. Fixing $s, t$, we have $p_{s, t}(x, y, s, t)=0$ for all $x, y$. This easily implies that for this $s, t$, we have a factorization

$$
p_{s, t}(x, y, z, w)=q(x, y)(t z-s w)
$$

for some $q(x, y) \in R_{2,0}$ (which depends on our fixed $s, t$ ). This factorization shows that in $\mathbb{P}^{5}, p_{s, t}$ gives a point of the Segre variety $Y$. It follows that $\varphi$ gives a map

$$
\varphi: \mathbb{P}^{1} \rightarrow W(p) \cap Y
$$

This proves that $W(p) \cap Y$ is infinite. Part (1) of the theorem follows immediately.

It remains to consider part (2) of the theorem. For this, we need to study $W(p) \cap Y$ when the intersection is infinite. If $W(p) \cap Y$ has dimension $>1$, then we must have $W(p) \subset Y$ since $W(p)$ is a plane. This gives

$$
\mathbb{P}^{2} \simeq W(p) \subset Y \simeq \mathbb{P}^{2} \times \mathbb{P}^{1}
$$

Since all morphisms $\mathbb{P}^{2} \rightarrow \mathbb{P}^{1}$ are constant, it follows that $W(p)$ corresponds to $\mathbb{P}^{2} \times\left\{\left(c_{1}, c_{2}\right)\right\} \subset \mathbb{P}^{2} \times \mathbb{P}^{1} \simeq Y$. When we think of $Y$ as consisting of reducible polynomials in $R_{2,1}$, we see that every polynomial in $W(p)$ is divisible by the fixed linear form $\ell=c_{1} z+c_{2} w$. This is impossible since $p_{0}, p_{1}, p_{2}$ have no common zeros on $\mathbb{P}^{1} \times \mathbb{P}^{1}$. Hence $\operatorname{dim}(W(p) \cap Y)>1$ can't occur.

For the rest of the proof we will assume that $\operatorname{dim}(W(p) \cap Y)=1$. We first show that in this case, $W(p) \cap Y$ is contained in a conic in $W(p) \simeq \mathbb{P}^{2}$. To see why, recall that $Y=Q_{1} \cap Q_{2} \cap Q_{3}$, where $Q_{1}, Q_{2}, Q_{3} \subset \mathbb{P}^{5}$ are the quadric hypersurfaces defined by the $2 \times 2$ minors of (4.1). By the above paragraph, we know that $W(p) \not \subset Y$, so that $W(p) \not \subset Q_{i}$ for at least one $i$. For this $i$, we have

$$
W(p) \cap Y \subset W(p) \cap Q_{i},
$$

where $W(p) \cap Q_{i}$ is a conic in $W(p)$. Thus $W(p) \cap Y$ lies in a conic in $W(p)$.

To complete the proof, we will show the following:

(a) $W(p) \cap Y$ is a smooth conic in $W(p)$.

(b) A suitable parametrization of this conic gives a nonzero element of $\operatorname{Syz}(p)_{2,3}$.

(c) For $n \geq 3$, every element of $\operatorname{Syz}(p)_{2, n}$ is obtained from the element of $\operatorname{Syz}(p)_{2,3}$ from (b) by multiplication by a unique polynomial in $R_{0, n-3}$. 
Note that part (2) of the theorem follows immediately from (b) and (c).

We will prove (a), (b), and (c) simultaneously by studying curves in $W(p) \cap Y$ parametrized by $\mathbb{P}^{1}$. Suppose that

$$
\phi: \mathbb{P}^{1} \rightarrow W(p) \cap Y,
$$

is a parametrization which, when regarded as a map to $\mathbb{P}^{5}$, is given by polynomials of degree $N$. This means that $\phi^{*} \mathcal{O}_{\mathbb{P}^{5}}(1)$ is $\mathcal{O}_{\mathbb{P}^{1}}(N)$. However, we can also view $\phi$ as a map to $Y \simeq \mathbb{P}^{2} \times \mathbb{P}^{1}$ given by

$$
\phi(s, t)=\left(r_{0}(s, t), r_{1}(s, t), r_{2}(s, t)\right) \times\left(h_{0}(s, t), h_{1}(s, t)\right) \in \mathbb{P}^{2} \times \mathbb{P}^{1},
$$

where $r_{0}, r_{1}, r_{2}$ have degree $\alpha$ and are relatively prime, and $h_{0}, h_{1}$ have degree $\beta$ and are relatively prime. It follows that

$$
\phi^{*} \mathcal{O}_{\mathbb{P}^{2} \times \mathbb{P}^{1}}(1,1) \simeq \mathcal{O}_{\mathbb{P}^{1}}(\alpha+\beta) .
$$

Since $\mathcal{O}_{\mathbb{P}^{5}}(1)$ pulls back to $\mathcal{O}_{\mathbb{P}^{2} \times \mathbb{P}^{1}}(1,1)$ under the Segre embedding, it follows that $\mathcal{O}_{\mathbb{P}^{5}}(1)$ pulls back to $\mathcal{O}_{\mathbb{P}^{1}}(\alpha+\beta)$ under $\phi$. Comparing this to our earlier description of the pullback, we conclude that

$$
N=\alpha+\beta .
$$

We next show that neither $\alpha$ nor $\beta$ can be zero. Suppose, for example, that $\alpha=0$. This implies that in $\mathbb{P}\left(R_{2,1}\right)$, we have

$$
\phi(s, t)=g(x, y)\left(h_{0}(s, t) z+h_{1}(s, t) w\right) .
$$

where $g(x, y) \in R_{2,0}$ does not depend on $s, t$. This gives a two-dimensional subspace of $R_{2,1}$ whose elements are all divisible by $g(x, y)$. However, $\phi$ parametrizes a curve in $W(p)=\mathbb{P}(\widetilde{W}(p))$, where $\widetilde{W}(p)=\operatorname{Span}\left(p_{0}, p_{1}, p_{2}\right) \subset$ $R_{2,1}$. It follows that $\widetilde{W}(p)$ has a two-dimensional subspace whose elements are all divisible by $g(x, y)$. Since $p_{0}, p_{1}, p_{2}$ have no common zeros on $\mathbb{P}^{1} \times \mathbb{P}^{1}$, an easy argument shows that any two linearly independent elements of $\widetilde{W}(p)$ must be relatively prime. This shows that the two-dimensional subspace constructed above can't exist. This contradiction proves that $\alpha>0$, and $\beta>0$ follows similarly.

Now we can prove (a). We showed above that $W(p) \cap Y$ is contained in a conic in $W(p)$. If the conic is not smooth, then it is a union of two lines, so that $W(p) \cap Y$ contains a line $\ell$. This gives a parametrization (4.3) with $N=1$. Since $N=\alpha+\beta$ with $\alpha$ and $\beta$ positive, this is impossible. It follows that the conic must be smooth and hence must equal $W(p) \cap Y$. Thus (a) is proved.

We now turn to (b). Since $W(p) \cap Y$ is a smooth conic, we can find a parametrization (4.3) with $N=2$. Since $N=\alpha+\beta$ with $\alpha, \beta>0$, we must have $\alpha=\beta=1$. In $\mathbb{P}\left(R_{2,1}\right)$, (4.4) can be written

$$
\phi(s, t)=\left(r_{0}(s, t) x^{2}+r_{1}(s, t) x y+r_{2}(s, t) y^{2}\right)\left(h_{0}(s, t) z+h_{1}(s, t) w\right),
$$

where $r_{0}, r_{1}, r_{2}, h_{0}, h_{1}$ have degree 1 in $s, t$. We also know that $h_{0}, h_{1}$ are relatively prime, which means that they are linearly independent in $R_{0,1}$. 
Thus, by reparametrizing $\phi$, we may assume that $h_{0}(s, t)=t$ and $h_{1}(s, t)=$ $-s$. Hence

$$
\phi(s, t)=\left(r_{0}(s, t) x^{2}+r_{1}(s, t) x y+r_{2}(s, t) y^{2}\right)(t z-s w)
$$

in $\mathbb{P}\left(R_{2,1}\right)$. Since $\phi$ gives a curve in $W$, we can also write $\phi(s, t)$ in the form

$$
B_{0}(s, t) p_{0}(x, y, z, w)+B_{1}(s, t) p_{1}(x, y, z, w)+B_{2}(s, t) p_{2}(x, y, z, w) .
$$

These expressions for $\phi(s, t)$ are the same. The first vanishes when $(s, t)=$ $(z, w)$, so that the same is true for the second. Hence

$$
B_{0}(z, w) p_{0}(x, y, z, w)+B_{1}(z, w) p_{1}(x, y, z, w)+B_{2}(z, w) p_{2}(x, y, z, w)=0 .
$$

Since $B_{0}(z, w), B_{1}(z, w), B_{2}(z, w)$ have degree 2 in $z, w$, it follows that we get a nonzero element of $\operatorname{Syz}(p)_{2,3}$. This proves (b).

For (c), suppose that we have a nonzero syzygy in $\operatorname{Syz}(p)_{2, n}$. As in the proof of part (1) of the theorem, we can write the syzygy in the form (4.2), namely

$$
h(z, w)\left(A_{0}(z, w), A_{1}(z, w), A_{2}(z, w)\right),
$$

where $A_{0}, A_{1}, A_{2}$ are relatively prime. This gives a map $\mathbb{P}^{1} \rightarrow W(p) \cap Y$ defined by

$$
(s, t) \mapsto p_{s, t}(x, y, z, w):=A_{0}(s, t) p_{0}+A_{1}(s, t) p_{1}+A_{2}(s, t) p_{2}
$$

with the property that

$$
p_{s, t}(x, y, s, t)=0 .
$$

The image is the smooth conic $W(p) \cap Y$. Since $A_{0}, A_{1}, A_{2}$ are relatively prime and $W(p) \cap Y$ is parametrized by $B_{0}, B_{1}, B_{2}$ from (4.6) and (4.7), one can show that there are relatively prime polynomials $u(s, t), v(s, t)$ such that

$$
A_{i}(s, t)=B_{i}(u(s, t), v(s, t)), \quad i=0,1,2 .
$$

(We leave the details to the reader.) Combining this with (4.6) shows that

$$
\begin{aligned}
p_{s, t}= & \left(r_{0}(u(s, t), v(s, t)) x^{2}+r_{1}(u(s, t), v(s, t)) x y+r_{2}(u(s, t), v(s, t)) y^{2}\right) \times \\
& (v(s, t) z-u(s, t) w) .
\end{aligned}
$$

Then (4.8) tells us that

$$
v(s, t) s-u(s, t) t=0 .
$$

This equation easily implies that

$$
u(s, t)=s g(s, t) \quad \text { and } \quad v(s, t)=\operatorname{tg}(s, t) .
$$

Since $u(s, t)$ and $v(s, t)$ are relatively prime, it follows that $g(s, t)$ is a nonzero constant $c$. This in turn implies that

$$
p_{s, t}=\left(r_{0}(c s, c t) x^{2}+r_{1}(c s, c t) x y+r_{2}(c s, c t) y^{2}\right)(c t z-c s w)=c^{2} \phi(s, t) .
$$

Hence $\left(A_{0}, A_{1}, A_{2}\right)$ is a constant times $\left(B_{0}, B_{1}, B_{2}\right)$. Then (c) follows. 
One comment is that if $p_{0}, p_{1}, p_{2} \in R_{2,1}$ are allowed to be arbitrary, then $W(p)$ can be an arbitrary plane in $\mathbb{P}^{5}$. In particular, $W(p) \cap Y$ can be a singular conic or a double line. But once we require that $p_{0}, p_{1}, p_{2}$ have no common zeros on $\mathbb{P}^{1} \times \mathbb{P}^{1}$, then $W(p) \cap Y$ is restricted to being finite or a smooth conic.

4.3. An Example. Here is an example to show that the non-generic behavior described in Theorem 4.1 can actually occur.

Example 4.3. Suppose that $p_{0}=x^{2} z, p_{1}=y^{2} w$ and $p_{2}=x^{2} w+y^{2} z$. One easily checks that these polynomials have no common zeros on $\mathbb{P}^{1} \times \mathbb{P}^{1}$. It is also easy to verify that

$$
w^{2} p_{0}+z^{2} p_{1}-z w p_{2}=0 .
$$

Thus $\left(w^{2}, z^{2},-z w\right)$ gives a nonzero element of $\operatorname{Syz}(p)_{2,3}$. As explained in the proof of the theorem, this is the special syzygy whose multiples by polynomials in $z, w$ account for the question marks in (3.4).

The corresponding curve $W(p) \cap Y \subset W$ is parametrized by

$$
\begin{aligned}
\varphi(s, t) & =t^{2} p_{0}+s^{2} p_{1}-s t p_{2} \\
& =t^{2} \cdot x^{2} z+s^{2} \cdot y^{2} w-s t\left(x^{2} w+y^{2} z\right) \\
& =\left(t x^{2}-s y^{2}\right)(t z-s w) .
\end{aligned}
$$

The third line shows that we have a curve in $W(p) \cap Y$, and the first line makes it clear that it is a smooth conic.

\section{Hilbert Functions and Generators}

Tools: Hilbert functions, functoriality

5.1. Hilbert Functions. Theorem 4.1 tells us that triples $\left(p_{0}, p_{1}, p_{2}\right) \in$ $R_{2,1}^{3}$ with no common zeros on $\mathbb{P}^{1} \times \mathbb{P}^{1}$ come in two flavors, generic and non-generic. It is now easy to determine the Hilbert function of $\operatorname{Syz}(p)$.

The Hilbert function of a finitely generated bigraded $R$-module $M$ is defined by

$$
H_{M}(m, n):=\operatorname{dim}_{k} M_{m, n} .
$$

For example, the Hilbert function of $R=k[x, y, z, w]$ is

$$
H_{R}(m, n)= \begin{cases}(m+1)(n+1) & m, n \geq-1 \\ 0 & \text { otherwise }\end{cases}
$$

Here is the Hilbert function of the syzygy module.

Proposition 5.1. Let $\operatorname{Syz}(p)$ be the syzygy module on $p_{0}, p_{1}, p_{2}$. 
(1) In the generic case, $\operatorname{Syz}(p)$ has Hilbert function

$$
H_{\mathrm{Syz}(p)}(m, n)= \begin{cases}3(m-3)(n-1)-(m-5)(n-2) & m \geq 5, n \geq 2 \\ m-5 & m \geq 6, n=1 \\ 2(n-2) & m=3, n \geq 3 \\ 3(n-1)+n-2 & m=4, n \geq 3 \\ 0 & \text { otherwise. }\end{cases}
$$

(2) In the non-generic case, $\operatorname{Syz}(p)$ has has Hilbert function

$$
H_{\text {Syz }(p)}(m, n)= \begin{cases}3(m-3)(n-1)-(m-5)(n-2) & m \geq 5, n \geq 2 \\ m-5 & m \geq 6, n=1 \\ (m-1)(n-2) & m=2,3, n \geq 3 \\ 3(n-1)+n-2 & m=4, n \geq 3 \\ 0 & \text { otherwise. }\end{cases}
$$

Proof. When $m \geq 5, n \geq 2$, Lemma 2.1] and Proposition 3.2 give the exact sequence

$$
0 \longrightarrow R_{m-6, n-3} \longrightarrow R_{m-4, n-2}^{3} \longrightarrow \mathrm{Syz}(p)_{m, n} \longrightarrow 0 .
$$

This easily implies that $H_{\mathrm{Syz}(p)}(m, n)=3(m-3)(n-1)-(m-5)(n-2)$ for these $(m, n)$ 's.

When $n=1, m=3$, or $m=4$, the formulas for $H_{\mathrm{Syz}(p)}(m, n)$ follow similarly from Lemma 2.1 and Proposition 3.2 When $m=2$ and $n \geq 3$, Theorem 4.1 tells us that $H_{\mathrm{Syz}(p)}(2, n)$ equals 0 in the generic case and $n-2$ in the non-generic case.

The remaining cases are all 0 by the zeros in (3.4).

Using Proposition 5.1 and the exact sequence

$$
0 \longrightarrow \operatorname{Syz}(p) \longrightarrow R(-2,-1)^{3} \longrightarrow I \longrightarrow 0,
$$

it is easy to determine the Hilbert function of the ideal $I=\left\langle p_{0}, p_{1}, p_{2}\right\rangle$. Hence $I$ also has two Hilbert functions, depending on whether $p_{0}, p_{1}, p_{2}$ are generic or non-generic.

5.2. Generators of the Syzygy Module. We next determine degrees of the minimal generators of the syzygy module in the generic and non-generic cases.

Proposition 5.2. Let $\operatorname{Syz}(p)$ be the syzygy module on $p_{0}, p_{1}, p_{2}$.

(1) In the generic case, $\operatorname{Syz}(p)$ has six minimal generators: one in degree $(6,1)$, three in degree $(4,2)$, and two in degree $(3,3)$.

(2) In the non-generic case, $\operatorname{Syz}(p)$ has five minimal generators: one in degree $(6,1)$, three in degree $(4,2)$, and one in degree $(2,3)$. 
Proof. First assume that $p_{0}, p_{1}, p_{2}$ are generic. Then the question marks in (3.4) are all zeros. Furthermore, we have the three Koszul syzygies in degree $(4,2)$, which generate $\operatorname{Syz}(p)$ in degree $(m, n)$ for all bullets in (3.4).

It remains to consider the boxes. When $n=1$, we get the horizontal line of boxes in (3.4). We proved that $\operatorname{Syz}(p)_{m, 1}$ has dimension $m-5$ for $m \geq 6$ using

$$
\begin{aligned}
\operatorname{Syz}(p)_{m, 1}={ }^{\prime \prime} E_{2}^{0,1}(m, n) & =H^{1}(m-6,-2) \\
& \simeq H^{0}\left(\mathbb{P}^{1}, \mathcal{O}_{\mathbb{P}^{1}}(m-6)\right) \otimes H^{1}\left(\mathbb{P}^{1}, \mathcal{O}_{\mathbb{P}^{1}}(-2)\right) \\
& \simeq k[x, y]_{m-6} .
\end{aligned}
$$

The key point is that everything here-spectral sequences, the Künneth formula, etc. - is functorial. In particular, the above isomorphisms are compatible with multiplication by homogeneous polynomials in $k[x, y]$. This gives the commutative diagram

$$
\begin{array}{ccc}
\operatorname{Syz}(p)_{6,1} \otimes k[x, y]_{m-6} & \simeq & k[x, y]_{0} \otimes k[x, y]_{m-6} \\
\downarrow & & \downarrow \\
\operatorname{Syz}(p)_{m, 1} & \simeq & k[x, y]_{m-6} .
\end{array}
$$

where the vertical maps are multiplication. Since the vertical map on the right is an isomorphism, the same is true for the map on the left. This shows that the non-Koszul syzygy in degree $(6,1)$ generates $\operatorname{Syz}(p)_{m, 1}$ for $m \geq 6$.

A similar argument shows that the two non-Koszul syzygies in degree $(3,3)$ generate $\operatorname{Syz}(p)_{3, n}$ for $n \geq 3$. For $m=4$, this argument also shows that

$$
{ }^{\prime \prime} E_{2}^{0,1}(4,3) \otimes k[z, w]_{n-3} \longrightarrow{ }^{\prime \prime} E_{2}^{0,1}(4, n)
$$

is surjective. Recall from Proposition 3.2 that ${ }^{\prime \prime} E_{2}^{0,1}(4,3)$ is one-dimensional, corresponding to one non-Koszul syzygy in degree $(4,3)$. Using Lemma 2.1 we see that $\operatorname{Syz}(p)_{4, n}$ is generated by the Koszul syzygies in degree $(4,2)$ plus the non-Koszul syzygy in degree $(4,3)$.

It remains to show that the non-Koszul syzygy in degree $(4,3)$ can be generated using the non-Koszul syzygies in degree $(3,3)$. For this, we first observe that

$$
\begin{aligned}
& { }^{\prime \prime} E_{2}^{0,1}(3,3) \simeq H^{1}\left(\mathbb{P}^{1}, \mathcal{O}_{\mathbb{P}^{1}}(-3)\right) \otimes H^{0}\left(\mathbb{P}^{1}, \mathcal{O}_{\mathbb{P}^{1}}(0)\right) \\
& { }^{\prime \prime} E_{2}^{0,1}(4,3) \simeq H^{1}\left(\mathbb{P}^{1}, \mathcal{O}_{\mathbb{P}^{1}}(-2)\right) \otimes H^{0}\left(\mathbb{P}^{1}, \mathcal{O}_{\mathbb{P}^{1}}(0)\right) .
\end{aligned}
$$

Furthermore, since the duality (3.2) is functorial, the map

$$
H^{1}\left(\mathbb{P}^{1}, \mathcal{O}_{\mathbb{P}^{1}}(-3)\right) \otimes k[x, y]_{1} \longrightarrow H^{1}\left(\mathbb{P}^{1}, \mathcal{O}_{\mathbb{P}^{1}}(-2)\right)
$$

is dual to

$$
H^{0}\left(\mathbb{P}^{1}, \mathcal{O}_{\mathbb{P}^{1}}(0)\right) \otimes k[x, y]_{1} \longrightarrow H^{0}\left(\mathbb{P}^{1}, \mathcal{O}_{\mathbb{P}^{1}}(1)\right) .
$$

This map is $k[x, y]_{0} \otimes k[x, y]_{1} \rightarrow k[x, y]_{1}$, which is obviously injective. Hence the dual map (5.2) is surjective, which by the functorality of the isomorphisms (5.1) means that the non-Koszul syzygy in degree $(4,3)$ comes from the syzygies in degree $(3,3)$. This completes the proof in the generic case. 
In the non-generic case, we get a non-Koszul syzygy in degree $(2,3)$. The proof of Theorem 4.1 shows that it generates $\operatorname{Syz}(p)_{2, n}$ for $n \geq 3$. Multiplying this syzygy by $x$ and $y$ gives two syzygies in degree $(3,3)$. It is easy to see that these are linearly independent and hence generate $\operatorname{Syz}(p)_{3,3}$. Then the argument in the generic case shows that this syzygy, together with the Koszul syzygies in degree $(4,2)$ and the non-Koszul syzygy in degree $(6,1)$, generate $\operatorname{Syz}(p)$.

5.3. The Free Resolution of $I$. If we combine the generators of the syzygy module described in Proposition 5.2 with the exact sequence

$$
0 \longrightarrow \operatorname{Syz}(p) \longrightarrow R(-2,-1)^{3} \longrightarrow I \longrightarrow 0,
$$

then we can start to build a minimal free resolution of the ideal $I=$ $\left\langle p_{0}, p_{1}, p_{2}\right\rangle$. In the generic case, we get

$$
\begin{array}{cc} 
& R(-6,-1) \\
\oplus & R(-4,-2)^{3} \longrightarrow R(-2,-1)^{3} \longrightarrow I \longrightarrow 0 \\
& R(-3,-3)^{2}
\end{array}
$$

while in the non-generic case, the resolution begins

$$
\begin{aligned}
& R(-6,-1) \\
& \oplus \\
& \cdots \longrightarrow R(-4,-2)^{3} \longrightarrow R(-2,-1)^{3} \longrightarrow I \longrightarrow 0 . \\
& \oplus \\
& R(-2,-3)
\end{aligned}
$$

In Section 17 we will show how to complete these partial resolutions.

Recall that once we have a minimal free resolution, it is straightforward to determine the Hilbert function. Since we already know the Hilbert function (Proposition 5.1), one might hope to reconstruct the minimal free resolution from the Hilbert function. Unfortunately, this is not possible in general, since different minimal free resolutions can have the same Hilbert function.

Example 5.3. Let $S=k[x]$, graded in the usual way. Then consider the graded $S$-modules $M_{1}$ and $M_{2}$ defined by the exact sequences

$$
0 \longrightarrow S(-2) \stackrel{\left[x^{2}\right]}{\longrightarrow} S \longrightarrow M_{1} \longrightarrow 0
$$

and

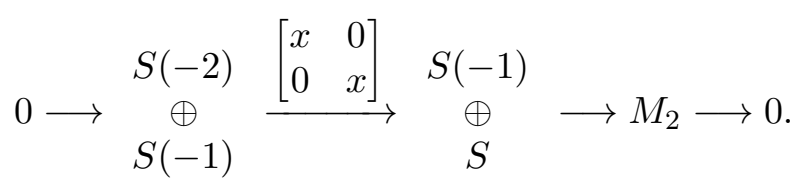

These sequences give the minimal free resolutions of $M_{1}$ and $M_{2}$. One easily sees that $M_{1}$ and $M_{2}$ have the same Hilbert function, even though the free resolutions are very different. 
We will see in Section 1 that in our situation, there is a one-to-one correspondence between Hilbert functions and minimal free resolutions.

\section{Explicit Non-Koszul Syzygies}

Tools: determinants, resultants

6.1. Formulas for Syzygies. Koszul syzygies have the nice property that they are given by explicit formulas; namely, they are generated by the columns of the $3 \times 3$ matrix in (1.1). The goal of this section is to give explicit formulas for the non-Koszul syzygies.

Suppose that for $i=0,1,2$ we have polynomials

$$
p_{i}=a_{i} x^{2} z+b_{i} x y z+c_{i} y^{2} z+d_{i} x^{2} w+e_{i} x y w+f_{i} y^{2} w
$$

with no common zeros on $\mathbb{P}^{1} \times \mathbb{P}^{1}$. We will describe a simple method for constructing one explicit nonzero syzygy in degree $(6,1)$ and two linearly independent syzygies in degree $(3,3)$.

We start with a straightforward lemma.

Lemma 6.1. Suppose that there exist bihomogeneous polynomials $g, h$ such that

$$
p_{i}=A_{i} g+B_{i} h, \quad i=0,1,2,
$$

and let $C_{i j}=A_{i} B_{j}-A_{j} B_{i}$. Then $C:=\left(C_{12}, C_{20}, C_{01}\right)$ is a syzygy on $p_{0}, p_{1}, p_{2}$.

Proof. The determinant

$$
\operatorname{det}\left[\begin{array}{ccc}
p_{0} & A_{0} & B_{0} \\
p_{1} & A_{1} & B_{1} \\
p_{2} & A_{2} & B_{2}
\end{array}\right]
$$

vanishes, since by our assumption (6.2), its first column equals $g$ times its second column plus $h$ times the third. The Laplace expansion of this determinant along the first column gives the identity

$$
p_{0}\left(A_{1} B_{2}-A_{2} B_{1}\right)+p_{1}\left(A_{2} B_{0}-A_{0} B_{2}\right)+p_{2}\left(A_{0} B_{1}-A_{1} B_{0}\right)=0 .
$$

This proves that $C$ is a syzygy on $p_{0}, p_{1}, p_{2}$.

This lemma can be used to construct Koszul syzygies as follows.

Example 6.2. If we let $g=1, h=0$, and $A_{i}=p_{i}$ in (6.2), then Lemma 6.1 gives a syzygy for any choice $B_{0}, B_{1}, B_{2}$ of bihomogeneous polynomials of the same degree. One easily sees that the resulting syzygy is

$$
B_{0}\left(0,-p_{2}, p_{1}\right)+B_{1}\left(-p_{2}, 0, p_{0}\right)+B_{2}\left(p_{1},-p_{0}, 0\right) .
$$

This is an arbitrary Koszul syzygy. In other words, letting $\left(B_{0}, B_{1}, B_{2}\right)=$ $(1,0,0),(0,1,0)$, and $(0,0,1)$ gives generators of the submodule of Koszul syzygies. 
We now construct a syzygy of degree $(6,1)$. Recall that this will involve polynomials of degree $(4,0)$ since $(6,1)=(4,0)+(2,1)$. Using (6.1), we write each $p_{i}$ in the form

$$
p_{i}=A_{i}(x, y) z+B_{i}(x, y) w, \quad i=0,1,2,
$$

with

$$
A_{i}(x, y)=a_{i} x^{2}+b_{i} x y+c_{i} y^{2}, \quad B_{i}(x, y)=d_{i} x^{2}+e_{i} x y+f_{i} y^{2}
$$

of degree $(2,0)$. Thus the syzygy $C$ from Lemma (6.1) involves polynomials of degree $(4,0)$.

Proposition 6.3. Let $A_{i}$ and $B_{i}$ be as above Then the syzygy $C$ from Lemma 6.1 is a non-Koszul syzygy in $\operatorname{Syz}(p)_{6,1}$.

Proof. Taking $g=z, h=w$ in the previous lemma, we know that $C$ is indeed a syzygy. Note that all $C_{i j}$ have degree $(4,0)$ and so they cannot be a multiple of any $p_{i}$. So, to prove that the syzygy is non-Koszul we only need to show that $C$ is nonzero. If this were so, then the matrix

$$
\left[\begin{array}{lll}
A_{0} & A_{1} & A_{2} \\
B_{0} & B_{1} & B_{2}
\end{array}\right]
$$

would have rank at most 1 . Hence there would be $(\alpha, \beta) \in \mathbb{A}^{2} \backslash\{(0,0)\}$ such that $\alpha\left(A_{0}, A_{1}, A_{2}\right)+\beta\left(B_{0}, B_{1}, B_{2}\right)=0$. Then the three polynomials $p_{0}, p_{1}, p_{2}$ would vanish at any point of the form $(x, y, \alpha, \beta)$, a contradiction.

We next construct two syzygies of degree $(3,3)$. These will involve polynomials of degree $(1,2)$ since $(3,3)=(1,2)+(2,1)$. Write the polynomials $p_{0}, p_{1}, p_{2}$ from (6.1) in the form

$$
p_{i}=C_{i}(z, w) x^{2}+D_{i}(z, w) x y+E_{i}(z, w) y^{2}, \quad i=0,1,2,
$$

where $C_{i}, D_{i}, E_{i}$ are homogeneous linear forms in $z, w$, i.e., bihomogeneous polynomials of degree $(0,1)$. Explicitly, $C_{i}(z, w)=a_{i} z+d_{i} w, D_{i}(z, w)=$ $b_{i} z+e_{i} w$, and $E_{i}(z, w)=c_{i} z+f_{i} w$. If we take $g=x, h=y^{2}$ in Lemma 6.1 and write

$$
p_{i}=\left(C_{i}(z, w) x+D_{i}(z, w) y\right) x+E_{i}(z, w) y^{2}, \quad i=0,1,2,
$$

then we get a syzygy $C^{(1)}$. On the other hand, if we take $g=x^{2}, h=y$ and write

$$
p_{i}=C_{i}(z, w) x^{2}+\left(D_{i}(z, w) x+E_{i}(z, w) y\right) y, \quad i=0,1,2,
$$

then Lemma 6.1 gives a second syzygy $C^{(2)}$. Note that $C^{(1)}$ and $C^{(2)}$ lie in $\operatorname{Syz}(p)_{3,3}$.

Proposition 6.4. The non-Koszul syzygies in degree $(3,3)$ are spanned by the syzygies $C^{(1)}$ and $C^{(2)}$ constructed above. 
Proof. Since $\operatorname{dim}_{k} \operatorname{Syz}(p)_{3,3}=2$ and there are no Koszul syzygies in degree $(3,3)$, it is enough to show that $C^{(1)}$ and $C^{(2)}$ are linearly independent.

We first observe that $C^{(1)} \neq 0$. To see why, note that $C^{(1)}=0$ implies that

$$
\begin{aligned}
& \left(C_{1} x+D_{1} y\right) E_{2}-\left(C_{2} x+D_{2} y\right) E_{1}=0 \\
& \left(C_{2} x+D_{2} y\right) E_{0}-\left(C_{0} x+D_{0} y\right) E_{2}=0 \\
& \left(C_{0} x+D_{0} y\right) E_{1}-\left(C_{1} x+D_{1} y\right) E_{0}=0
\end{aligned}
$$

The first equation can be rewritten as

$$
\left(C_{1} E_{2}-C_{2} E_{1}\right) x-\left(D_{1} E_{2}-D_{2} E_{1}\right) y=0
$$

and similarly for the other equations. Hence these equations imply the vanishing of certain $2 \times 2$ minors of

$$
\left[\begin{array}{ccc}
C_{0} & C_{1} & C_{2} \\
D_{0} & D_{1} & D_{2} \\
E_{0} & E_{1} & E_{2}
\end{array}\right]
$$

It follows easily that (6.4) does not have maximal rank, which by (6.3) implies that $p_{0}, p_{1}, p_{2}$ are linearly dependent. Yet we observed in Section 4 that $p_{0}, p_{1}, p_{2}$ are linearly independent since they have no common zeros on $\mathbb{P}^{1} \times \mathbb{P}^{1}$. Thus $C^{(1)} \neq 0$.

If $C^{(1)}$ and $C^{(2)}$ were linearly dependent, then $C^{(2)}$ would be a constant times $C^{(1)}$. Since $C^{(1)}$ is formed from the $2 \times 2$ minors of

$$
\left[\begin{array}{ccc}
C_{0} x+D_{0} y & C_{1} x+D_{1} y & C_{2} x+D_{2} y \\
E_{0} & E_{1} & E_{2}
\end{array}\right],
$$

it lies in the kernel of this matrix, and similarly $C^{(2)}$ lies in the kernel of

$$
\left[\begin{array}{ccc}
C_{0} & C_{1} & C_{2} \\
D_{0} x+E_{0} y & D_{1} x+E_{1} y & D_{2} x+E_{2} y
\end{array}\right] .
$$

If $C^{(2)}$ were a multiple of $C^{(1)}$, then it is straightforward to show that $C^{(1)}$ would lie in the kernel of (6.4). Since $C^{(1)} \neq 0$, we conclude that (6.4) would not have maximal rank. As above, this is impossible, and linear independence follows.

Propositions 6.3 and 6.4 give explicit formulas for one non-Koszul syzygy of degree $(6,1)$ and two linearly independent non-Koszul syzygies of degree $(3,3)$. When combined with the three Koszul syzygies of degree $(4,2)$, Proposition 5.2 shows that we have explicit formulas for the generators of the syzygy module $\operatorname{Syz}(p)$ in the generic case. In Section 7 we will use the internal structure of these explicit formulas to determine the free resolution of $I=\left\langle p_{0}, p_{1}, p_{2}\right\rangle$ in the generic case. 
6.2. The Non-Generic Case. As we have already seen, non-Koszul syzygies in degree $(2,3)$ occur only when $p_{0}, p_{1}, p_{2}$ are non-generic. They correspond to the case when the linear span of our polynomials intersects the variety of $R_{2,0} \times R_{0,1}$ factorizable polynomials in a smooth conic.

One way to proceed is to compute the syzygies $C^{(1)}, C^{(2)}$ of degree $(3,3)$. The analysis of Section 5 shows that these are multiples of a syzygy of degree $(2,3)$ by linear forms in $x, y$. Then we get the desired syzygy of degree $(2,3)$ by taking the GCD of $C^{(1)}$ and $C^{(2)}$.

Example 6.5. We revisit Example 4.3, where $p_{0}=x^{2} z, p_{1}=y^{2} w$ and $p_{2}=x^{2} w+y^{2} z$. One computes that

$$
\begin{aligned}
& C^{(1)}=\left(-x w^{2},-x z^{2}, x z w\right) \\
& C^{(2)}=\left(-y w^{2},-y z^{2}, y z w\right) .
\end{aligned}
$$

These are multiples (by $-x$ and $-y$ respectively) of the syzygy $\left(w^{2}, z^{2},-z w\right)$ described in Example 4.3

In general, we can use this method to construct the non-Koszul syzygy in degree $(2,3)$ when $W(p) \cap Y$ is a smooth conic. We first state an easy consequence of Theorem 4.1 that we will also need in Section $\mathbf{7}$

Lemma 6.6. In the non-generic case, we may assume after a change of basis in $W(p)=\mathbb{P}\left(\operatorname{Span}\left(p_{0}, p_{1}, p_{2}\right)\right)$ that $p_{0}, p_{1}, p_{2}$ are of the form $p_{i}=g_{i} l_{i}, i=$ $0,1,2$, where $g_{i} \in R_{(2,0)}$ and $l_{i} \in R_{(0,1)}$. Furthermore, we may assume that $g_{2}=g_{0}+g_{1}, l_{0}=z, l_{1}=w$, and $l_{2}=z+w$. Moreover, $\left\langle g_{0}, g_{1}\right\rangle$ and $\left\langle p_{0}, p_{1}\right\rangle$ are complete intersections.

Proof. As in the proof of Theorem 4.1, we can parametrize $W(p) \cap Y$ by

$$
\phi(s, t)=\left(r_{0}(s, t) x^{2}+r_{1}(s, t) x y+r_{2}(s, t) y^{2}\right)(t z-s w)
$$

where $r_{i}(s, t)$ is linear in $s, t$ (see (4.6) ). Note that any three points on a smooth conic define the same $\mathbb{P}^{2}$, since $n+1$ points on a rational normal curve of degree $n$ are in linearly general position by [Harris, p. 10]. Hence we can replace our original $p_{0}, p_{1}, p_{2}$ with

$$
p_{0}=\phi(0,1), p_{1}=\phi(-1,0), p_{2}=\phi(-1,1),
$$

i.e., $p_{i}=g_{i} l_{i}$ with $l_{0}=z, l_{1}=w, g_{0}=r_{0}(0,1) x^{2}+r_{1}(0,1) x y+r_{2}(0,1) y^{2}, g_{1}=$ $r_{0}(-1,0) x^{2}+r_{1}(-1,0) x y+r_{2}(-1,0) y^{2}$ and $l_{2}=z+w, g_{2}=g_{0}+g_{1}$, by the linearity of the $r_{i}$. If $\left\langle g_{0}, g_{1}\right\rangle$ is not a complete intersection, they share a zero $\left(x_{0}, y_{0}\right) \in \mathbb{P}^{1}$, from which $\left(x_{0}, y_{0}, z, w\right)$ would be a common zero of $p_{0}, p_{1}, p_{2}$ for any value of $(z, w)$, a contradiction. It easily follows that $\left\langle p_{0}, p_{1}\right\rangle$ is also a complete intersection.

Changing to a different basis of $W(p)=\mathbb{P}\left(\operatorname{Span}\left(p_{0}, p_{1}, p_{2}\right)\right)$ induces an isomorphism of the corresponding syzygy modules. So, we assume that our non-generic polynomials $p_{0}, p_{1}, p_{2}$ are as in the statement of Lemma 6.6] and 
we keep the notation of the proof. One can compute that in this case the syzygies $C^{(1)}$ and $C^{(2)}$ of Proposition 6.4 are given by

$$
\begin{aligned}
& C^{(1)}=-\left(m_{13} x+m_{23} y\right)(w(z+w), z(z+w),-z w) \\
& C^{(2)}=-\left(m_{12} x+m_{13} y\right)(w(z+w), z(z+w),-z w),
\end{aligned}
$$

where $m_{i j}$ is the $2 \times 2$ minor formed using columns $i$ and $j$ of the rank 2 matrix

$$
\left[\begin{array}{ccc}
r_{0}(0,1) & r_{1}(0,1) & r_{2}(0,1) \\
r_{0}(-1,0) & r_{1}(-1,0) & r_{2}(-1,0)
\end{array}\right]
$$

Here, $C^{(1)}$ and $C^{(2)}$ are common multiples of $(w(z+w), z(z+w),-z w)$, which is easily seen to be a syzygy on (6.5) since $r_{i}(-1,1)=r_{i}(0,1)+r_{i}(-1,0)$. This gives the desired element of $\operatorname{Syz}(p)_{2,3}$.

6.3. Resultants. We end this section with a "computational" translation of our hypothesis that $p_{0}, p_{1}, p_{2}$ have no common zeros on $\mathbb{P}^{1} \times \mathbb{P}^{1}$. This condition is precisely described by the non-vanishing of the multihomogeneous unmixed resultant $\operatorname{Res}_{(2,1)}\left(p_{0}, p_{1}, p_{2}\right)$. As described in Cox-Little-O'Shea, Ch. 7], we can regard this resultant as an irreducible polynomial $\operatorname{Res}_{(2,1)} \in$ $\mathbb{Z}\left[a_{0}, \ldots, f_{2}\right]$, where the variables $a_{0}, \ldots, f_{2}$ represent the 18 coefficients of $p_{0}, p_{1}, p_{2}$ in (6.1).

The polynomial $\operatorname{Res}_{(2,1)}$ can be expressed most efficiently as a determinant. Of the known ways of doing this, the smallest can be obtained using the methods in Dickenstein-Emiris. Consider the dehomogenizations $P_{i}(x, z):=p_{i}(x, 1, z, 1)$. Thus

$$
P_{i}=a_{i} x^{2} z+b_{i} x z+c_{i} z+d_{i} x^{2}+e_{i} x+f_{i} \in k[x, z], \quad i=0,1,2 .
$$

Let $X, Z$ be two new variables, and denote by $B$ the matrix

$$
B=\left[\begin{array}{ccc}
P_{0}(x, z) & P_{1}(x, z) & P_{2}(x, z) \\
P_{0}(X, z) & P_{1}(X, z) & P_{2}(X, z) \\
P_{0}(X, Z) & P_{1}(X, Z) & P_{2}(X, Z)
\end{array}\right]
$$

One can show that the expression

$$
b=\frac{1}{(x-X)(z-Z)} \operatorname{det}(B)
$$

is in fact a polynomial in $x, z, X$, and $a_{0}, \ldots, f_{2}$. It is also easy to see that $b$ has degree 3 in $X$ and degree 1 in $x$ and $z$. Thus $b$ has an expansion

$$
b(x, z, X)=\sum_{j=0}^{3} b_{0 j} X^{j}+b_{1 j} x X^{j}+b_{2 j} z X^{j}+b_{3 j} x z X^{j},
$$

where $b_{i j} \in \mathbb{Z}\left[a_{0}, \ldots, f_{2}\right]$ for $0 \leq i, j \leq 3$. The results of Dickenstein-Emiris] imply that $\operatorname{Res}_{(2,1)}=\operatorname{det}\left(b_{i j}\right)$. This compact expression is a polynomial of degree 12 in the 18 coefficients of $p_{0}, p_{1}, p_{2}$, whose expansion as a sum of monomials in $a_{0}, \ldots, f_{2}$ has 20,791 terms. 


\section{The Shape of the Minimal Free Resolutions}

Tools: computations, mapping cones, Hilbert-Burch theorem, liason

7.1. Computations. In Section 5.3, we determined the first syzygies of an ideal generated by three elements of $R_{(2,1)}$ with no common zeros on $\mathbb{P}^{1} \times \mathbb{P}^{1}$; the syzygies differ in the generic and non-generic cases. It is natural to ask about higher syzygies, and more generally about the minimal free resolution. Resolutions can be calculated by a computer, which we illustrate using Macaulay 2.

Example 7.1. For multigraded rings, Macaulay 2 requires weights which have a nonzero first entry. So we will alter the grading of $R$ a bit, creating a ring where $x, y$ have degree $(1,0)$ and $z, w$ have degree $(1,1)$. Hence $p_{0}, p_{1}, p_{2}$ have degree $(3,1)$ in this grading.

We return again to Example 4.3

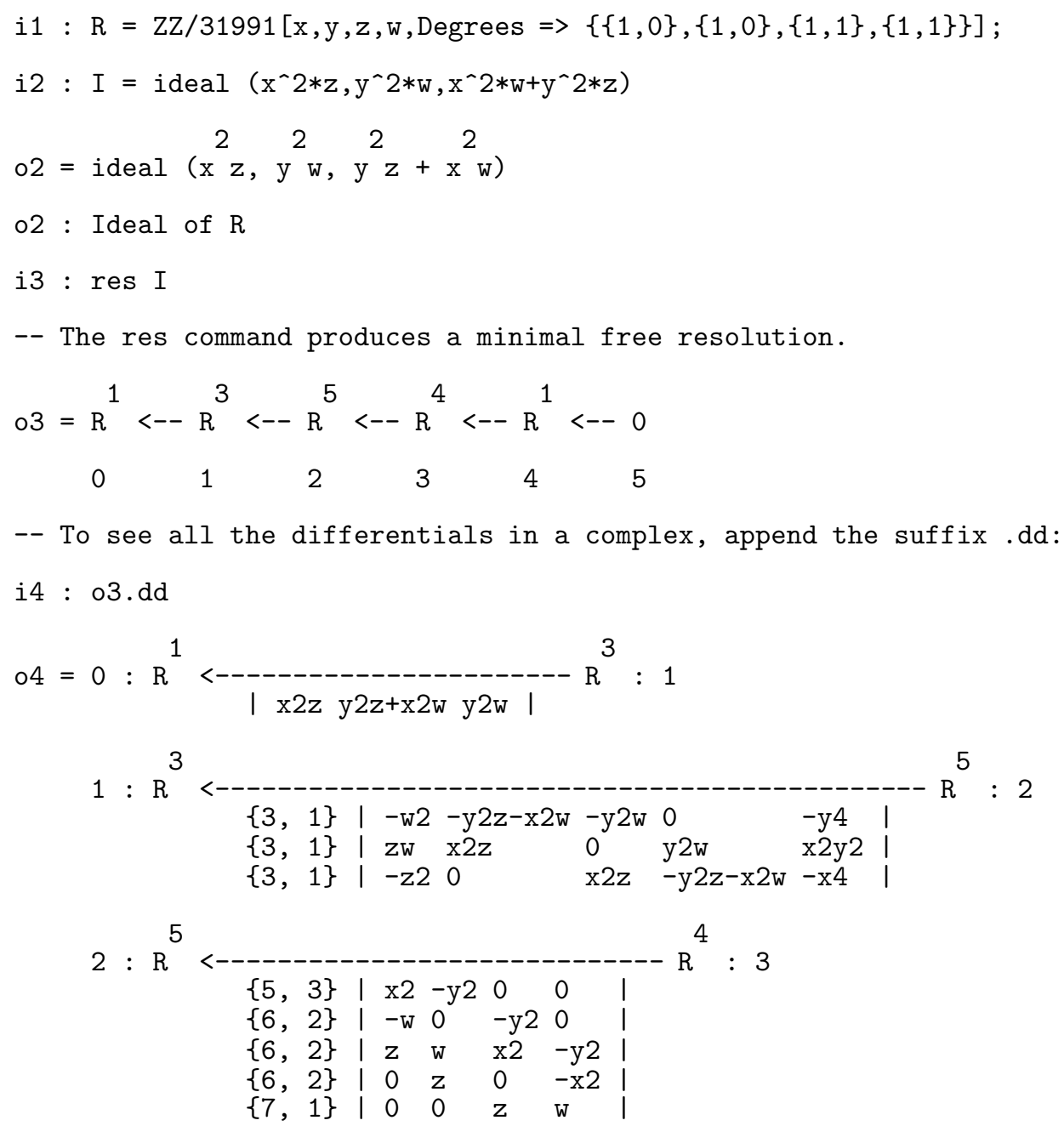




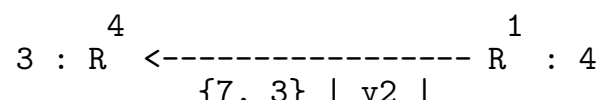

$$
\begin{aligned}
& \{7,3\} \mid \mathrm{x} 2 \text { । } \\
& \{8,2\} \mid-\mathrm{W} \\
& \{8,2\}|z| \\
& 4: R^{1}<\frac{0--0}{0}<: 5
\end{aligned}
$$

In this example, $p_{0}=x^{2} z, p_{1}=y^{2} z+x^{2} w, p_{2}=y^{2} w$, and the second, third and fourth columns of the matrix of the second differential correspond to the generators of the Koszul syzygies $k_{01}=\left(-p_{1}, p_{0}, 0\right), k_{02}=\left(-p_{2}, 0, p_{0}\right)$ and $k_{21}=\left(0, p_{2},-p_{1}\right)$. Also, calling $C_{1}, \ldots, C_{4}$ the columns of the matrix of the third differential (which generate the second syzygies), note that $y^{2} C_{1}+$ $x^{2} C_{2}=w C_{3}-z C_{4}=\left(0,-p_{2}, p_{1}, p_{0}, 0\right)$, yielding the second Koszul syzygy $\left(-p_{2}, p_{1}, p_{0}\right)$ of the first Koszul syzygies $-p_{2} k_{01}+p_{1} k_{02}+p_{0} k_{21}=0$.

Example 7.2. We now consider the generic case. We use the same setup as for Example 7.1, except that now we ask Macaulay 2 to resolve the ideal generated by 3 random elements of degree $(3,1)$.

We obtain the following resolution:

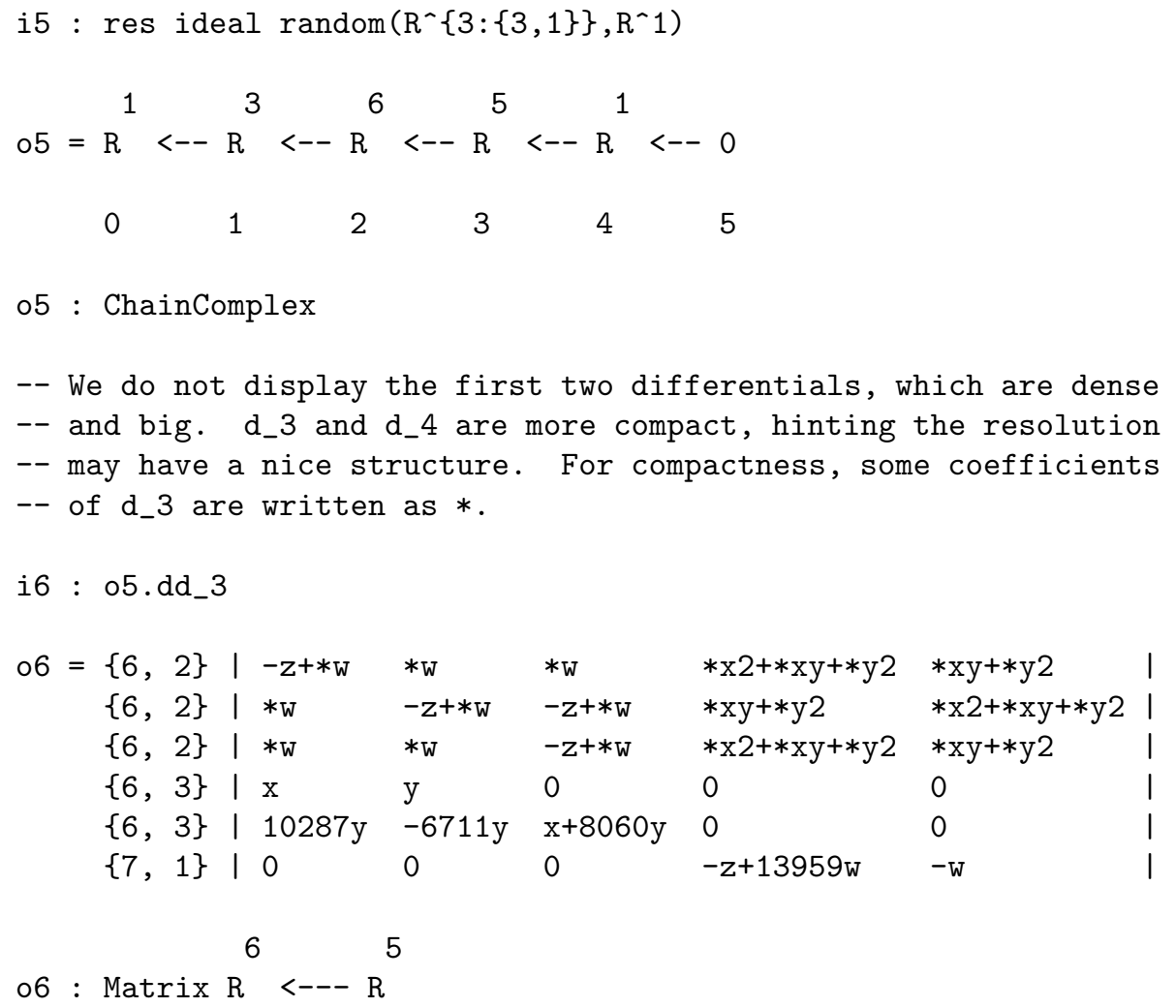




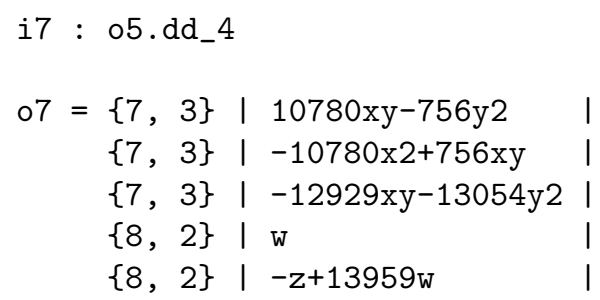

In Proposition 5.2 we learned that there are only two possible types of behavior for the first syzygies. In general, this tells us nothing about higher syzygies. However, the zeros in the third differentials in Examples 7.1] and 7.2 suggest that there may be some interesting structure in the resolutions. The intuition is that having a zero entry means that some syzygy involves only part of the previous syzygy module, so there may be a way to split things up. This is indeed the case, and we now discuss the tools we shall need.

7.2. The mapping cone. Let $R$ be a ring, $f \in R$ and $L$ an ideal of $R$. There is an exact sequence

$$
0 \longrightarrow(L+\langle f\rangle) / L \longrightarrow R / L \longrightarrow R /(L+\langle f\rangle) \longrightarrow 0 .
$$

The map $1 \mapsto f$ gives a surjection from $R$ to $(L+\langle f\rangle) / L$; the kernel of the map is simply $\{g \in R \mid f g \in L\}=(L: f)$. Notice that if $R$ is graded, $L$ is homogeneous, and $f \in R_{a}$, then we actually obtain a graded exact sequence:

$$
0 \longrightarrow R(-a) /(L: f) \stackrel{[f]}{\longrightarrow} R / L \longrightarrow R /(L+\langle f\rangle) \longrightarrow 0 .
$$

Suppose we have graded free resolutions $F_{\bullet}$ of $R(-a) /(L: f)$ and $G_{\bullet}$ of $R / L$ with $F_{0}=R(-a)$ and $G_{0}=R$. Then we have a commutative diagram:

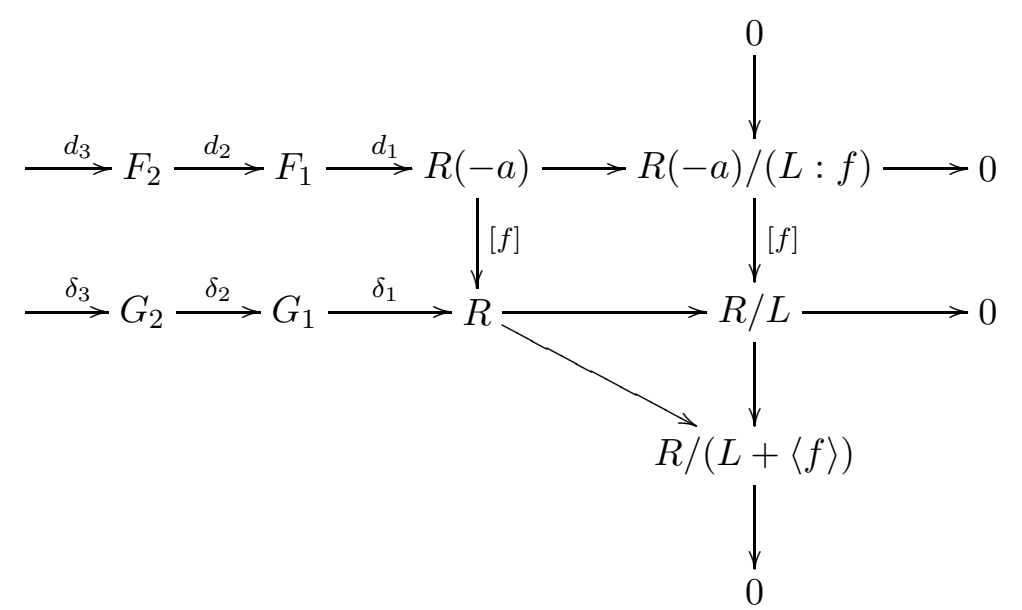

The rightmost column is exact by construction. Furthermore, an easy diagram chase shows that the diagonal map fits into an exact sequence

$$
R(-a) \oplus G_{1} \stackrel{[f] \oplus \delta_{1}}{\longrightarrow} R \longrightarrow R /(L+\langle f\rangle) \longrightarrow 0 .
$$

So we have the beginning of a free resolution for $R /(L+\langle f\rangle)$. 
Lemma 7.3. In the setting above, there is a map of complexes from $F_{\bullet}$ to $G_{\bullet}$ such that $H_{0}\left(F_{\bullet}\right) \rightarrow H_{0}\left(G_{\bullet}\right)$ is the map

$$
R(-a) /(L: f) \stackrel{[f]}{\longrightarrow} R / L .
$$

Proof. This is a standard result in homological algebra - see Eisenbud, Prop. A3.13], for example. You should review the argument to see that if the graded free resolutions $F_{\bullet}$ and $G_{\bullet}$ are given explicitly, then so is the map between them.

Now that we have a map $\psi_{\bullet}: F_{\bullet} \rightarrow G_{\bullet}$, the process of constructing a resolution for $R /(L+\langle f\rangle)$ is pretty simple. We already have the first two steps in the exact sequence

$$
R(-a) \oplus G_{1} \stackrel{[f] \oplus \delta_{1}}{\longrightarrow} R \longrightarrow R /(L+\langle f\rangle) \longrightarrow 0 .
$$

Let $M_{0}:=G_{0}=R$, and for all $i \geq 1$, define modules $M_{i}:=F_{i-1} \oplus G_{i}$, where $F_{0}=R(-a)$. Now define maps $M_{i} \stackrel{\partial_{i}}{\longrightarrow} M_{i-1}$ via $\partial_{1}:=[f] \oplus \delta_{1}$ for $i=1$ and

$$
\partial_{i}:=\left[\begin{array}{cc}
d_{i-1} & 0 \\
(-1)^{i-1} \psi_{i-1} & \delta_{i}
\end{array}\right]
$$

for $i \geq 2$. A straightforward computation shows that $\partial_{i} \circ \partial_{i-1}=0$, so that $\left(M_{\bullet}, \partial_{\bullet}\right)$ is a complex. In this notation, the first two steps of the resolution of $R /(L+\langle f\rangle)$ can be written as

$$
M_{1} \longrightarrow M_{0} \longrightarrow R /(L+\langle f\rangle) \longrightarrow 0 .
$$

Lemma 7.4. The complex $\left(M_{\bullet}, \partial_{\bullet}\right)$ is resolution of $R /(L+\langle f\rangle)$.

Proof. This can be proved by an explicit argument using the formula for $\partial_{i}$ and the fact that $F_{\bullet}$ and $G_{\bullet}$ are resolutions of $R(-a) /(L: f)$ and $R / L$ respectively. Alternatively, it is easy to see that there is a short exact sequence of complexes

$$
0 \longrightarrow G_{\bullet} \longrightarrow M_{\bullet} \longrightarrow F_{\bullet}[-1] \longrightarrow 0
$$

where $\left(F_{\bullet}[-1]\right)_{i}=F_{i-1}$. Using the corresponding long exact sequence in homology (see [Eisenbud, Prop. A3.15] or [Schenck, Thm. 8.1.4]) easily gives the desired result. It is a good exercise to work out the details of both proofs.

The complex $\left(M_{\bullet}, \partial_{\bullet}\right)$ is called the mapping cone. While it always gives a resolution, it need not be minimal; in particular, not all the entries of $\partial_{i}$ need to have positive degree. We illustrate the mapping cone with a pair of examples.

Example 7.5. The minimal free resolution of the quotient of a ring by an ideal generated by a regular sequence $f_{1}, \ldots, f_{n}$ is the Koszul complex of the $f_{i}$. This can be proved by induction using the mapping cone construction since the definition of a regular sequence implies that $\left(\left\langle f_{1}, \ldots, f_{k}\right\rangle: f_{k+1}\right)=$ $\left\langle f_{1}, \ldots, f_{k}\right\rangle$. See [Schenck, §3.3] for more details. 
Example 7.6. In $k[x, y]$, consider the ideal $L=\left\langle x^{2}, y^{2}\right\rangle$ and the element $f=x y$. It is easy to see that $(L: x y)=(x, y)$, so that $F_{\bullet}$ is a Koszul complex on $x, y$, and $G_{\bullet}$ is a Koszul complex on $x^{2}, y^{2}$. The resulting mapping cone resolution of $R /(L+\langle f\rangle)=R /\left\langle x^{2}, y^{2}, x y\right\rangle$ is therefore:

$$
0 \rightarrow R(-4) \rightarrow R(-4) \oplus R^{2}(-3) \rightarrow R^{3}(-2) \rightarrow R \rightarrow R /\left\langle x^{2}, y^{2}, x y\right\rangle \rightarrow 0 .
$$

However, the actual minimal resolution is:

$$
0 \longrightarrow R^{2}(-3) \longrightarrow R^{3}(-2) \longrightarrow R \longrightarrow R /\left\langle x^{2}, y^{2}, x y\right\rangle \longrightarrow 0,
$$

where the maps are given by:

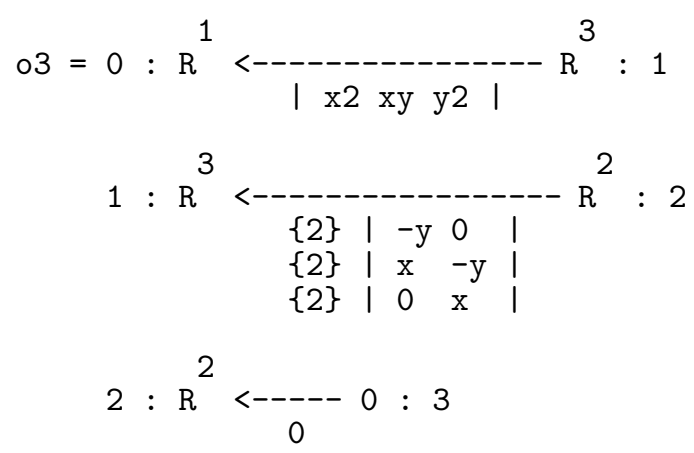

In Example 7.6, it is interesting to note that in the minimal resolution, the two-by-two minors of the matrix of first syzygies actually generate the ideal. This is no accident - it is guaranteed by the following classic result.

Theorem 7.7 (The Hilbert-Burch theorem). Let

$$
\mathcal{F}: 0 \longrightarrow F_{2} \stackrel{d_{2}}{\longrightarrow} F_{1} \stackrel{d_{1}}{\longrightarrow} R \longrightarrow R / L \longrightarrow 0
$$

be a complex where $F_{1}$ and $F_{2}$ are free. Then:

(1) If $\mathcal{F}$ is exact and $F_{1} \simeq R^{n}$, then $F_{2} \simeq R^{n-1}$ and there exists a nonzerodivisor a such that $L=a I_{n-1}\left(d_{2}\right)$, where $I_{n-1}\left(d_{2}\right)$ is the Fitting ideal generated by the $(n-1) \times(n-1)$ minors of $d_{2}$. Furthermore, the $i^{\text {th }}$ entry of $d_{1}$ is $(-1)^{i}$ a times the minor obtained from $d_{2}$ by deleting the $i^{\text {th }}$ row and the ideal $L$ has depth exactly two.

(2) Conversely, given any $(n-1) \times n$ matrix $d_{2}$ with depth $I_{n-1}\left(d_{2}\right) \geq 2$, and a nonzerodivisor a, the map $d_{1}$ obtained as in part (1) makes $\mathcal{F}$ into a free resolution of $R / L$, with $L=a I_{n-1}\left(d_{2}\right)$.

For a proof, we refer the reader to Eisenbud, Theorem 20.15]. The Hilbert-Burch theorem will prove to be useful below.

7.3. The Non-Generic Case. We now return to the problem of describing the free resolution of the ideal $I=\left\langle p_{0}, p_{1}, p_{2}\right\rangle$. We begin with the non-generic case. By Lemma 6.6 we may assume that

$$
p_{0}=g_{0} z, p_{1}=g_{1} w, p_{2}=g_{2} l_{2}=\left(g_{0}+g_{1}\right)(z+w),
$$


where $g_{0}, g_{1} \in R_{2,0}$ and $C=\left\langle g_{0} z, g_{1} w\right\rangle$ is a complete intersection. We shall obtain the entire free resolution of $I$ by taking two mapping cone resolutions. Consider the short exact sequence

$$
0 \longrightarrow R(-2,-1) /\left(C: g_{2} l_{2}\right) \stackrel{\left[g_{2} l_{2}\right]}{\longrightarrow} R / C \longrightarrow R / I \longrightarrow 0 .
$$

Since $C$ is a complete intersection, the free resolution of $R / C$ is a Koszul complex. To obtain a mapping cone resolution for $R / I$ (which is not, in general, a minimal resolution), we have to determine the ideal quotient $J=\left(C: g_{2} l_{2}\right)$.

The key to determining the ideal quotient is to use the fact that for any ideal $L=\left\langle f_{1}, \ldots, f_{k}\right\rangle$ and element $f$, an element $a$ is in $(L: f)$ if and only if there exist $a_{1}, \ldots, a_{k}$ such that $a f=\sum_{i=1}^{k} a_{i} f_{i}$, i.e. if and only if $\left(a_{1}, \ldots, a_{k},-a\right)$ is a syzygy on $\left(f_{1}, \ldots, f_{k}, f\right)$.

In our situation, Proposition 5.2 describes the degrees of the minimal generators of the syzygy module. The generators of degrees $(4,2)$ are the Koszul syzgyies

$$
\left(p_{1}, p_{0}, 0\right),\left(p_{2}, 0-p_{0}\right),\left(0, p_{2},-p_{1}\right),
$$

and by Proposition 6.3, the $2 \times 2$ minors of

$$
\left[\begin{array}{ccc}
g_{0} & 0 & g_{2} \\
0 & g_{1} & g_{2}
\end{array}\right]
$$

give the generator $\left(-g_{1} g_{2},-g_{0} g_{2}, g_{0} g_{1}\right)$ in degree $(6,1)$. Also, we saw in Section 6.2 that in the situation of Lemma [6.6. the generator in degree $(2,3)$ is the syzygy $(w(z+w), z(z+w),-z w)$. By the previous paragraph, it follows that $J=\left(I: g_{2} l_{2}\right)$ is given by

$$
J=\left\langle z w, g_{0} z, g_{1} w, g_{0} g_{1}\right\rangle .
$$

We need to find the resolution of this ideal, and we do it with (yet another!) mapping cone. First, we'll let $K$ denote the ideal $\left\langle z w, g_{0} z, g_{1} w\right\rangle$, so $J=K+\left\langle g_{0} g_{1}\right\rangle$. Consider the short exact sequence

$$
0 \longrightarrow R(-4,0) /\left(K: g_{0} g_{1}\right) \stackrel{\left[g_{0} g_{1}\right]}{\longrightarrow} R / K \longrightarrow R / J \longrightarrow 0 .
$$

From our choice of $\left\{g_{0} z, g_{1} w\right\}$ as a complete intersection, it follows that the depth of $K$ is at least two. Since the maximal minors of

$$
\left[\begin{array}{cc}
g_{0} & g_{1} \\
-w & 0 \\
0 & -z
\end{array}\right]
$$

generate $K$, the second part of Theorem 7.7 implies that we have a HilbertBurch resolution:

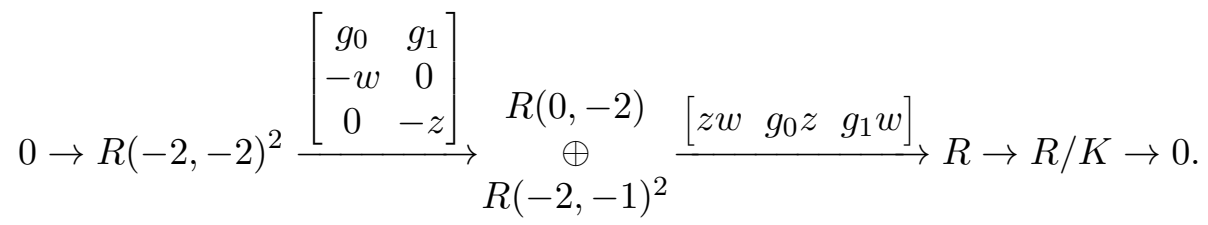


Now we need to compute the free resolution of $\left(K: g_{0} g_{1}\right)$. Observe that both $z$ and $w$ are in the quotient and generate the entire maximal ideal in $k[z, w]$. Any element of $K$ has degree at least one in these variables, and $g_{0} g_{1}$ has degree zero in these variables. Thus, any element of $\left(K: g_{0} g_{1}\right)$ has degree at least one in $z, w$, and so $z$ and $w$ must generate the whole ideal quotient. In other words, $\left(K: g_{0} g_{1}\right)=\langle z, w\rangle$

Since $\{z, w\}$ is a regular sequence, the resolution of $R(-4,0) /\left(K: g_{0} g_{1}\right)$ is a Koszul complex. When we combine this with the above Hilbert-Burch resolution for $R / K$ as in (7.1), we obtain the diagram:

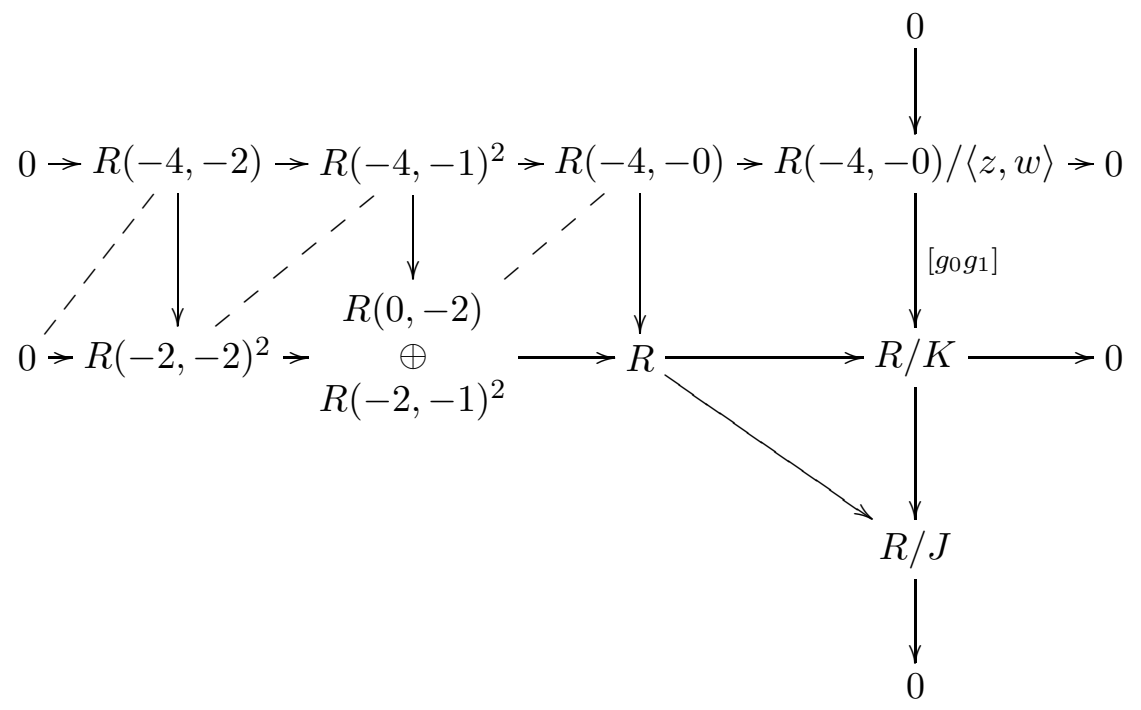

The mapping cone is constructed by taking the direct sum of modules connected by dashed lines in the diagram. This gives a free resolution for $R / J$ as follows:

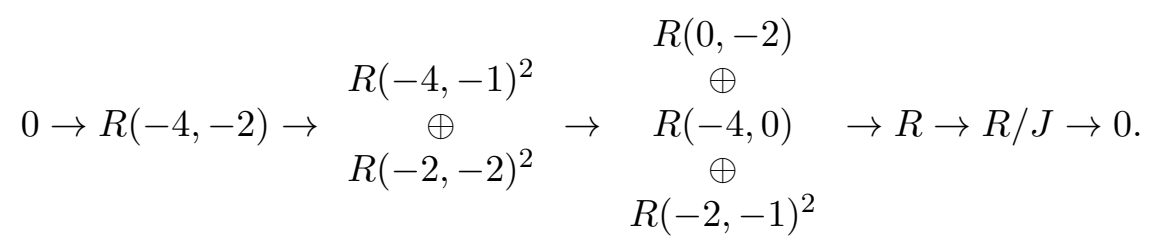

In fact, there is no overlap between the degree shifts, and so in fact the mapping cone resolution is minimal.

We are now in a position to construct a mapping cone resolution for $I$. Rather surprisingly, it, too, turns out to be a minimal free resolution. Recall that for $R / I$, we have the exact sequence

$$
0 \longrightarrow R(-2,-1) / J \stackrel{\left[g_{2} l_{2}\right]}{\longrightarrow} R / C \longrightarrow R / I \longrightarrow 0 .
$$

where $C=\left\langle p_{0}, p_{1}\right\rangle=\left\langle g_{0} z, g_{1} w\right\rangle$ and $J=\left(C: p_{2}\right)=\left(C: g_{2} l_{2}\right)$. Thus, if we take the Koszul resolution of $R / C$ and tensor the above resolution of $R / J$ with $R(-2,-1)$, we get a mapping cone diagram similar to (7.2) for $R / I$. We recommend that you write this out carefully. You will see that there is 
no overlap between degrees at consecutive steps of the resolution. Hence, the mapping cone resolution is minimal. Putting it all together, we have proved the following theorem.

Theorem 7.8. In the non-generic case, the minimal free resolution of the ideal $I=\left\langle p_{0}, p_{1}, p_{2}\right\rangle$ is given by:

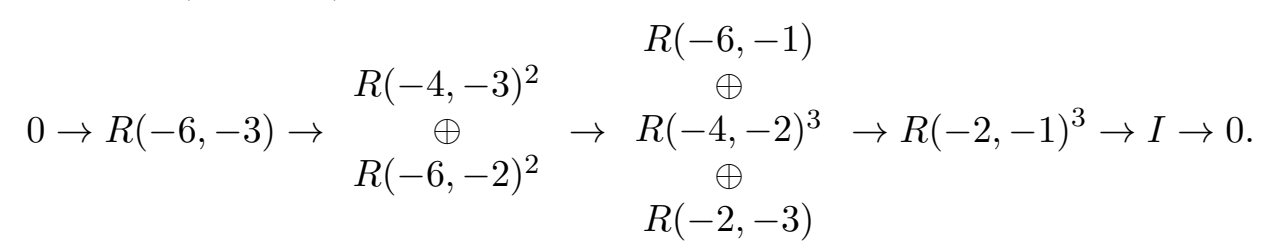

7.4. The Generic Case. Next, we tackle the generic case. It turns out that, just as in the non-generic case, we can obtain the free resolution from an iterated mapping cone construction. We again make use of the explicit formulas for the non-Koszul syzygies given in Section 6.2. This will give explicit generators for the ideal quotient. By unwinding the internal structure of these generators, we can use the same techniques to construct the minimal free resolution.

Theorem 7.9. In the generic case, the minimal free resolution of the ideal $I=\left\langle p_{0}, p_{1}, p_{2}\right\rangle$ is given by:

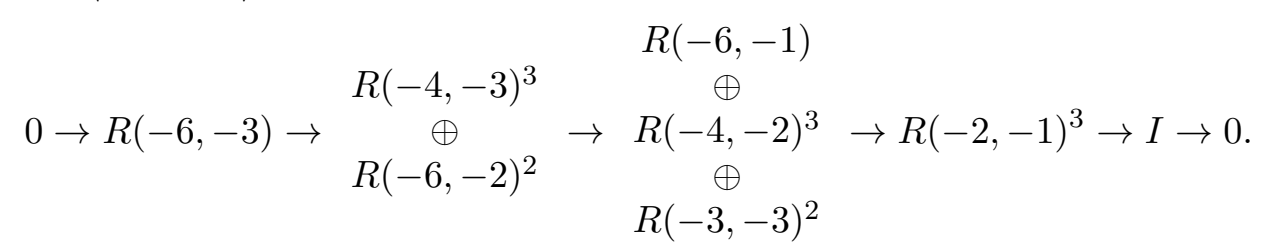

Proof. As in the non-generic case, we begin by choosing generators so that $I=\left\langle p_{0}, p_{1}, p_{2}\right\rangle$ with $C=\left\langle p_{0}, p_{1}\right\rangle$ a complete intersection. This is possible since two polynomials define a complete intersection, unless they share a common factor. On $\mathbb{P}^{1} \times \mathbb{P}^{1}$, such a common factor defines a curve of degree $(a, b)$, which would meet $\mathbf{V}\left(p_{2}\right)$ in $2 b+a$ points (see Hartshorne, Example V.1.4.3]), a contradiction to the assumption that $I$ has no common zeros. So there exists an exact sequence

$$
0 \longrightarrow R(-2,-1) /\left(C: p_{2}\right) \stackrel{\left[p_{2}\right]}{\longrightarrow} R / C \longrightarrow R / I \longrightarrow 0 .
$$

Proposition 5.2 gives the degrees of the generators of the syzygy module $\operatorname{Syz}(p)$, and as in the non-generic case, this gives the degrees of generators of $\left(C: p_{2}\right)$. Hence $\left(C: p_{2}\right)$ has two generators $p_{0}, p_{1}$ of degree $(2,1)$ corresponding to Koszul syzygies, two generators $k_{1}, k_{2}$ of degree $(1,2)$, and a generator $g$ of degree $(4,0)$.

Proposition 6.4 gives explicit descriptions for the generators $k_{1}, k_{2}$. If we write

$$
\begin{aligned}
& p_{0}=C_{0} x^{2}+D_{0} x y+E_{0} y^{2} \\
& p_{1}=C_{1} x^{2}+D_{1} x y+E_{1} y^{2},
\end{aligned}
$$


then we have

$$
k_{1}=\operatorname{det}\left[\begin{array}{ll}
C_{0} x+D_{0} y & E_{0} \\
C_{1} x+D_{1} y & E_{1}
\end{array}\right], \quad k_{2}=\operatorname{det}\left[\begin{array}{ll}
C_{0} & D_{0} x+E_{0} y \\
C_{1} & D_{1} x+E_{1} y
\end{array}\right] .
$$

To understand $\left(C: p_{2}\right)=\left\langle p_{0}, p_{1}, k_{1}, k_{2}, g\right\rangle$, we will use $K=\left\langle p_{0}, p_{1}, k_{1}, k_{2}\right\rangle$ and $(K: g)$. A check shows that the $3 \times 3$ minors of the matrix:

$$
\phi=\left[\begin{array}{ccc}
E_{1} & -D_{1} & C_{1} \\
E_{0} & -D_{0} & C_{0} \\
x & y & 0 \\
0 & x & y
\end{array}\right]
$$

generate $K$. Since the ideal generated by $\left\{p_{0}, p_{1}\right\}$ is a complete intersection, $\left\{p_{0}, p_{1}\right\}$ is a regular sequence, hence the depth of $K$ is at least two. This means that we have a Hilbert-Burch resolution, so $\phi$ is injective and we have a resolution

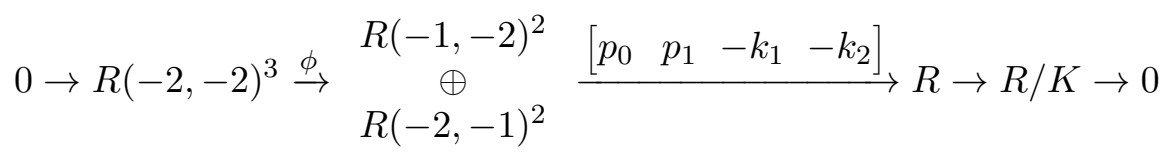

Since $\left(C: p_{2}\right)=K+\langle g\rangle$, we get a mapping cone resolution of $R /\left(C: p_{2}\right)$ (again, possibly non-minimal) from resolutions of $R / K$ (given above) and $R(-4,0) /(K: g)$ (still to be determined).

To study $(K: g)$, recall from Proposition 6.3 that if we write

$$
p_{0}=A_{0} z+B_{0} w \quad \text { and } \quad p_{1}=A_{1} z+B_{1} w,
$$

then $g=A_{0} B_{1}-A_{1} B_{0}$. This makes it easy to see that $z, w \in(K: g)$. From here on, everything works as in the non-generic case: check that $(K: g)=\langle z, w\rangle$, and construct a mapping cone resolution for $R /\left(C: p_{2}\right)$, which is forced to be minimal from degree considerations. By (7.3), the free resolutions of $R(-2,-1) /\left(C: p_{2}\right)$ and $R / C$ give a mapping cone resolution for $R / I$, which is also forced by degree considerations to be a minimal free resolution.

7.5. Minimal Resolutions. One point we want to emphasize again is that we were lucky that the resolutions resulting from the mapping cone construction were minimal. In practice, one usually has to "prune" the resolution before obtaining a minimal one. The paper Migliore-Miró-Roig gives some nice examples of how this is done. Finally, the fact that we had only three generators need not imply that the resolution will be simple; as Bruns shows in Bruns, essentially any resolution arises as the resolution of a three generated ideal.

7.6. Liason. The strategy of understanding $I=\left\langle p_{0}, p_{1}, p_{2}\right\rangle$ by applying the mapping cone construction to $C=\left\langle p_{0}, p_{1}\right\rangle$ and $J=\left(C: p_{2}\right)$ goes back to the classic paper Peskine-Szpiro of Peskine and Szpiro. In general, ideals $I$ 
and $J$ of codimension $c$ in a polynomial ring $R$ are said to be directly linked if there is a regular sequence $f_{1}, \ldots, f_{c}$ contained in $R$ such that

$$
J=\left(\left\langle f_{1}, \ldots, f_{c}\right\rangle: I\right) \text { and } I=\left(\left\langle f_{1}, \ldots, f_{c}\right\rangle: J\right) .
$$

When $R / I$ is Cohen-Macaulay, then one can use the mapping cone construction and duality to transform a free resolution of $R / I$ into a free resolution of $R / J$. See, for example, Eisenbud, Exercise 21.23]. An introduction to linkage (also called liason) can be found in Migliore.

In our situation, Bermejo and Gimenez observed that the symmetry of (7.4) fails; in particular (writing $\left.C=\left\langle p_{0}, p_{1}\right\rangle\right)$ we have $J=(C: I)$, but a computer check shows $I \neq(C: J)$. However, in the generic case, we proved that $(K: J)=(K: g)=\langle z, w\rangle$, and Bermejo and Gimenez noted that $K:\langle z, w\rangle=J$. So we recover the symmetry (7.4), though not quite as liason since $K$ is not a complete intersection. While the usual notion of liason (using a complete intersection) is a bit too restrictive for our situation, there are more general notions of liason (see Artin-Nagata or Huneke-Ulrich) which may be useful in understanding this symmetry.

\section{The implicitization PRoblem in GeOMETRIC MODELing}

A central problem in geometric modeling is to find the implicit equations for a curve or surface defined by a rational map. For surfaces, the two most common situations are the images of parameterizations $\mathbb{P}^{1} \times \mathbb{P}^{1} \longrightarrow \mathbb{P}^{3}$ or $\mathbb{P}^{2} \longrightarrow \mathbb{P}^{3}$. Surfaces of the first type are called tensor product surfaces, and surfaces of the latter type are called triangular surfaces.

The implicitization problem involves some interesting commutative algebra. For example, implicitization can be recast as a problem in elimination theory, which allows one to use standard tools such as Gröbner bases or resultants. The surprise is that more sophisticated tools from commutative algebra are also being used, and syzygies play a leading role! A detailed survey of this area appears in Cox] we sketch some of the highlights below.

8.1. Moving Curves and Surfaces. In Sederberg-Chen, Sederberg and Chen introduced the method of moving curves and surfaces. For a curve parametrization $(a, b, c)$, a moving line that follows the parametrization is a element of the syzygy module on the generators of the ideal $I=\langle a, b, c\rangle$. The syzygy module of $I$ is free of rank two, and a Hilbert function computation shows that if $a, b, c$ are homogeneous of degree $n$ without common factors, then there is an $n$-dimensional vector space of moving lines of degree $n-1$. Write each moving line as

$$
A_{i}(s, t) x+B_{i}(s, t) y+C_{i}(s, t) z
$$

where the $x, y, z$ are placeholders, representing the fact that $A_{i} \cdot a+B_{i} \cdot b+$ $C_{i} \cdot c=0$. By collecting coefficients, we can write

$$
A_{i}(s, t) x+B_{i}(s, t) y+C_{i}(s, t) z=\sum_{j=0}^{n-1} L_{i j}(x, y, z) s^{j} t^{n-1-j} .
$$


A main theorem of Cox-Sederberg-Chen is that the determinant of the $n \times n$ matrix of the $L_{i j}$ is a power of the implicit equation for the image.

For a surface parametrization given by $(a, b, c, d)$, a moving plane that follows the parametrization is an element of the syzygy module on the generators of the ideal $I=\langle a, b, c, d\rangle$, and a moving quadric that follows the parametrization is an element of the syzygy module on the generators of $I^{2}$. The moving surface method of Cox-Goldman-Zhang requires knowing that a syzygy of the form

$$
\left(c_{1} a+c_{2} b+c_{3} c+c_{4} d\right) a+\left(c_{5} b+c_{6} c+c_{7} d\right) b+\left(c_{8} c+c_{9} d\right) c=0
$$

comes from the Koszul complex when $a, b, c, d$ have no common zeros. In the case of $\mathbb{P}^{2}$ (i.e., when $a, b, c, d$ are homogeneous polynomials), this is proved by observing that $a, b, c$ form a regular sequence, so that every syzygy comes from the Koszul complex. For $\mathbb{P}^{1} \times \mathbb{P}^{1}$ (i.e., when $a, b, c, d$ are bihomogeneous polynomials), the Koszul complex is not exact in all bidegrees, but by vanishing theorems for cohomology and arguments similar to those explained in our first sections, it can be seen that the sequence is exact in the bidegree of interest.

The main result of Cox-Goldman-Zhang involves parameterizations without base points. When base points are allowed, Busé-Cox-D'Andrea shows that for $\mathbb{P}^{2}$, the moving surface method of Cox-Goldman-Zhang applies when the base points are local complete intersections. This is also true for $\mathbb{P}^{1} \times \mathbb{P}^{1}$, by Adkins-Hoffman-Wang. The proofs in Busé-Cox-D'Andrea use results about the regularity of $I$ and $I^{2}$; in a similar way, the proofs in Adkins-Hoffman-Wang use results about bigraded regularity.

Other directions of recent research are [Chen-Cox-Liu, which uses a special case of the Serre conjecture to conclude that syzygy modules are always free for affine surface parameterizations, and the use of approximation complexes (Busé-Jouanolou, Busé-Chardin) to study implicitization and moving surfaces. The results above suggest that the interaction between commutative algebra and implicitization is unusually rich.

8.2. Group Actions. While the tools of commutative algebra can be more complicated in the bigraded case, there are sometimes other tools that can be helpful. In the case of a generic tensor product surface of degree $(2,1)$ in $\mathbb{P}^{3}$, the recent paper Elkadi-Galligo-Lê uses group actions to analyze the geometry of the resulting surface. Here is a quick sketch of their results. Given four generic polynomials $p_{0}, p_{1}, p_{2}, p_{3}$ of degree $(2,1)$, one can use group actions by GL(4) and GL(2) $\times$ GL(2) to reduce to the case

$$
p_{0}=x^{2} z, p_{1}=(x-y)^{2}(z-w), p_{2}=(x-A y)^{2}(z-B w), p_{3}=y^{2} w,
$$

where $A$ and $B$ are parameters determined by certain cross ratios. Then Elkadi, Galligo and Lê show how to obtain the implicit equation of the surface in terms of a certain $4 \times 4$ matrix, and prove that the surface is singular along a twisted cubic. 
In our situation, we have three polynomials $p_{0}, p_{1}, p_{2}$ of degree $(2,1)$. Similar to the previous paragraph, there is the action of GL(3) that replaces $p_{0}, p_{1}, p_{2}$ with linear combinations, and second, the usual action of $\mathrm{GL}(2) \times \mathrm{GL}(2)$ on $\mathbb{P}^{1} \times \mathbb{P}^{1}$. In our non-generic case, we used these actions in Lemma 6.6 to put $p_{0}, p_{1}, p_{2}$ in a normal form.

In our generic case, we know that $W(p)=\operatorname{Span}\left(p_{0}, p_{1}, p_{2}\right)$ meets $Y \simeq$ $\mathbb{P}^{2} \times \mathbb{P}^{1}$ in finitely many points. Since $Y \subset \mathbb{P}^{5}$ has degree three, the nicest case is when $W(p)$ meets $Y$ in three distinct points. When this happens, we can pick $p_{0}, p_{1}, p_{2}$ to be these three points, and we get the following normal form:

$$
\begin{aligned}
& p_{0}=x(x-A y) z \\
& p_{1}=y(y-B x) w \\
& p_{2}=(x+y)(x-C y)(z+w) .
\end{aligned}
$$

As one can easily guess, the resultant in Section 6.3 is simply

$$
\operatorname{Res}_{(2,1)}=C(1+B)(B C-1)(1+A)(A-C)(A B-1) .
$$

It is easy to check that the components of the syzygy $C^{(1)}\left(\operatorname{resp} . C^{(2)}\right)$ from Section [6] have a common linear factor $B x-y$ (resp. $-x+A y)$ if and only if $A=B C+B+C-1$. Thus, we are in the non-generic case precisely when this equality holds. However, it may happen that $W(p)=\operatorname{Span}\left(p_{0}, p_{1}, p_{2}\right)$ meets $Y$ in a double point and a simple point. Consider

$$
\begin{aligned}
& p_{0}=x(x-A y) z \\
& p_{1}=y(y-B x) z+x(x-A y) w \\
& p_{2}=(x+y)(x-C y)(z+w),
\end{aligned}
$$

where $(1-A B)(A+1)(A-C) C \neq 0$. In this case, one can show that $W(p) \cap Y$ consists of only two points, $p_{0}$ and $p_{2}$, where $p_{0}$ has multiplicity two. One can also check that the line through $p_{0}$ and $p_{1}$ lies in the tangent space to $Y$ at $p_{0}$, which shows that the intersection $W(p) \cap Y$ is not transversal at $p_{0}$.

It follows that there is not a single "normal form" that covers all cases in which $p_{0}, p_{1}, p_{2}$ have no common roots on $\mathbb{P}^{1} \times \mathbb{P}^{1}$, even in our generic case when $W(p) \cap Y$ is finite.

\section{ACKNOWLEDGEMENTS}

Evidence for this work was provided by many computations done using Macaulay 2. Macaulay 2 is available at the URL

$$
\text { http://www.math.uiuc.edu/Macaulay2/ }
$$

We also thank Isabel Bermejo and Philippe Gimenez for helpful comments on the mapping cone strategy and liason. Alicia Dickenstein was partially supported by UBACYT X042 and ANPCYT 03-6568, Argentina. Hal Schenck was partially supported by NSF DMS03-11142 and NSA MDA904-03010006. We are grateful to MSRI, where some of this work took place. 


\section{REFERENCES}

[Adkins-Hoffman-Wang] W. Adkins, J. W. Hoffman, and H. H. Wang, Equations of parametric surfaces with basepoints via syzygies, preprint, arXiv math.AG/0306195

[Aramova-Crona-De Negri] A. Aramova, K. Crona, and E. De Negri, Bigeneric initial ideals, diagonal subalgebras and bigraded Hilbert functions, J. Pure Appl. Algebra 150 (2000), 215-235.

[Artin-Nagata] M. Artin and M. Nagata, Residual intersections in Cohen-Macaulay rings, J. Math. Kyoto Univ. 12 (1972), 302-323.

[Bruns] W. Bruns, Jede endlich frei Auflösung ist freie Auflösung eines von drei Elementen erzeugten Ideals, J. Algebra 39 (1976), 429-439.

[Busé-Chardin] L. Busé and M. Chardin, Implicitizing rational hypersurfaces using approximation complexes, preprint, arXiv math.AG/030123.

[Busé-Cox-D'Andrea] L. Busé, D. Cox and C. D'Andrea. Implicitization of surfaces in $\mathbb{P}^{3}$ in the presence of base points, J. Algebra Appl. 2 (2003), 189-214.

[Busé-Jouanolou] L. Busé and J. P. Jouanolou, On the closed image of a rational map and the implicitization problem, J. Algebra 265 (2003), 312-357.

[Chen-Cox-Liu] F. Chen, D. Cox, and Y. Liu, The $\mu$-basis of a rational parametric surface, J. Symbolic Comput., to appear.

[Cox] D. Cox, Equations of parametric curves and surfaces via syzygies, Contemporary Mathematics 286, AMS, Providence, RI, (2001), 1-20.

[Cox-Goldman-Zhang] D. Cox, R. Goldman, and M. Zhang, On the validity of implicitization by moving quadrics for rational surfaces with no base points, J. Symbolic Comput. 29 (2000), 419-440.

[Cox-Little-O'Shea] D. Cox, J. Little, and D. O'Shea, Using Algebraic Geometry, second edition, Springer-Verlag, Berlin-Heidelberg-New York, 2004.

[Cox-Sederberg-Chen] D. Cox, T. Sederberg, and F. Chen, The moving line ideal basis of planar rational curves, Comput. Aided Geom. Design 15 (1998), 419-440.

[Dickenstein-Emiris] A. Dickenstein and I. Emiris, Multihomogeneous resultant formulae by means of complexes, J. Symbolic Comput. 34 (2003), 317-342.

[Dieudonné-Grothendieck] J. Dieudonné and A. Grothendieck, Éléments de Géométrie Algébrique III, Publications Mathématiques 17, IHES, Bures-sur-Yvette, 1963.

[Eisenbud] D. Eisenbud, Commutative Algebra with a view towards Algebraic Geometry, Springer-Verlag, Berlin-Heidelberg-New York, 1995.

[Elkadi-Galligo-Lê] M. Elkadi, A. Galligo, and T. H. Lê, Parametrized surfaces in $\mathbb{P}^{3}$ of bidegree $(1,2)$, Proceedings of the 2004 International Symposium on Symbolic and Algebraic Computation, ACM, New York, 2004, 141-148.

[Griffiths-Harris] P. Griffiths and J. Harris, Principles of Algebraic Geometry, Wiley, New York, 1978.

[Giuffrida-Maggioni-Ragusa] S. Giuffrida, R. Maggioni, and A. Ragusa, On the postulation of 0-dimensional subschemes on a smooth quadric, Pacific J. Math. 155 (1992), 251282.

[Há-Van Tuyl] H. Há and A. Van Tuyl, The regularity of points in multi-projective spaces, J. Pure Appl. Algebra 187 (2004), 153-167.

[Harris] J. Harris, Algebraic Geometry, A First Course, Springer-Verlag, Berlin-Heidelberg-New York, 1992.

[Hartshorne] R. Hartshorne, Algebraic Geometry, Springer-Verlag, Berlin-Heidelberg-New York, 1977.

[Hoffman-Wang] J. W. Hoffman and H. H. Wang, Castelnuovo-Mumford regularity in biprojective spaces, Advances in Geometry 4 (2004), 513-536.

[Huneke-Ulrich] C. Huneke and B. Ulrich, Residual intersections, J. reine ang. Math. 390 (1988), 1-20. 
[Maclagan-Smith] D. Maclagan and G. G. Smith, Multigraded Castelnuovo-Mumford Regularity, J. reine ang. Math. 571 (2004), 179-212.

[Maclagan-Thomas] D. Maclagan and R. Thomas, The toric Hilbert scheme of a rank two lattice is smooth and irreducible, J. Combin. Theory Ser. A 104 (2003), 29-48.

[Migliore] J. Migliore, Introduction to Liason Theory and Deficiency Modules, Progress in Math. 165, Birkhäuser, Basel-Berlin-Boston, 1998.

[Migliore-Miró-Roig] J. Migliore and R. M. Miró-Roig, On the minimal free resolution of $n+1$ general forms, Trans. Amer. Math. Soc. 355 (2003), 1-36.

[Peeva-Stillman1] I. Peeva and M. Stillman, Local equations for the toric Hilbert scheme, Adv. in Appl. Math. 25 (2000), 307-321.

[Peeva-Stillman2] I. Peeva and M. Stillman, Toric Hilbert schemes, Duke Math. J. 111 (2002), 419-449.

[Peskine-Szpiro] C. Peskine and L. Szpiro, Liaison des variétés algébriques, Invent. Math. 26 (1974), 271-302.

[Römer] T. Römer, Homological properties of bigraded algebras, Illinois J. Math. 45 (2001), 1361-1376.

[Schenck] H. Schenck, Computational Algebraic Geometry, Cambridge University Press, 2003.

[Sederberg-Chen] T. Sederberg and F. Chen, Implicitization using moving curves and surfaces, in Proceedings of SIGGRAPH 1995, ACM, 1995, 301-308.

[Sidman-Van Tuyl] J. Sidman and A. Van Tuyl, Multigraded regularity: syzygies and fatpoints, preprint, arXiv math.AC/0405247

[Stillman-Sturmfels-Thomas] M. Stillman, B. Sturmfels, and R. Thomas, Algorithms for the toric Hilbert scheme, in Computations in algebraic geometry with Macaulay 2, 179-214, Algorithms Comput. Math. 8, Springer, Berlin, 2002.

[Sturmfels] B. Sturmfels, Gröbner Bases and Convex Polytopes, University Lecture Series 8, AMS, 1996.

[Van Tuyl] A. Van Tuyl, The border of the Hilbert function of a set of points in $\mathbb{P}^{n_{1}} \times$ $\cdots \times \mathbb{P}^{n_{k}}$, J. Pure Appl. Algebra 176 (2002), 223-247.

David Cox

Department of Mathematics and Computer Science, Amherst College

Amherst, MA 01002-5000

dac@cs. amherst.edu

Alicia Dickenstein

Departamento de Matemática, F.C.E. y N., Universidad de Buenos Aires Cuidad Universitaria-Pabellón I, 1428 Buenos Aires, Argentina

alidick@dm.uba.ar

Hal Schenck

Department of Mathematics, Texas A\&M University

College Station, TX 77843

schenck@math.tamu .edu 